\title{
The Complexity of Finding Small Triangulations of Convex 3-Polytopes.
}

\author{
Alexander Below* Jesús A. De Loera ${ }^{\dagger}$ \\ Jürgen Richter-Gebert ${ }^{\ddagger}$
}

October 23, 2018

\begin{abstract}
The problem of finding a triangulation of a convex three-dimensional polytope with few tetrahedra is $N P$-hard. We discuss other related complexity results.
\end{abstract}

\section{Introduction}

A triangulation of a $d$-dimensional convex polytope $P$ is a set of $d$-simplices whose union is the polytope, their vertices are extreme points of $P$, and any two simplices in it intersect in a common (possibly empty) face. The size of a triangulation is the number of its full-dimensional simplices. In this paper we discuss the computational complexity of finding small size triangulations of a convex polytope. We discuss in particular the case of minimal triangulations, i.e. those with smallest possible size.

This geometric minimization problem arises, for example, in Algebra and Mathematical Programming. For example, minimal triangulations of the $d$-cube have been extensively studied (see references in 11, 15, 16]) due to connections with the simplicial approximation of fixed points of continuous maps (see [27]). Optimal size triangulations appear also in the polyhedral techniques in Algebraic Geometry [26. Understanding minimal triangulations of convex polytopes is related to the problem of characterizing the $f$-vectors of triangulations of balls and polytopes (see open problems in [8]). In fact, the study of minimal triangulations of topological balls also received attention due to its connections to data structures, in the calculation of rotation distance of binary trees [25].

\footnotetext{
*Institut für Theoretische Informatik, ETH-Zürich (below@inf.ethz.ch).

${ }^{\dagger}$ Dept. of Mathematics, Univ. of California-Davis (deloera@math.ucdavis.edu).

${ }^{\ddagger}$ Institut für Theoretische Informatik, ETH-Zürich (richter@inf.ethz.ch).
} 
The computational geometry literature has several papers interested in finding triangulations of optimal size 5, 13. In 1992 Bern and Eppstein asked whether there is a polynomial time algorithm to compute a minimal triangulation of a 3-dimensional convex polytope (open problem 12 in section 3.2 [7]). Our main result shows that, under the hypothesis $P \neq N P$, such an algorithm cannot exist:

Theorem 1.1 Given a convex 3-polytope $P$ with $n$ vertices and a positive integer $K$, deciding whether $P$ has a triangulation of size $K$ or less is an NPcomplete problem.

We have the following corollaries (the second result was recently obtained in [20] via a direct transformation to 3 -SAT):

Corollary 1.2 1. Finding a minimal-size triangulation of a convex polytope of any fixed dimension $d \geq 3$ is NP-hard. Clearly, the same holds when the dimension is not fixed.

2. Finding a minimal-size triangulation of the boundary of a convex polytope of any fixed dimension $d \geq 4$ is NP-hard. Clearly, the same holds when the dimension is not fixed.

Now we discuss the general structure and main ideas of the proof of Theorem 1.1.

We give a transformation to the Satisfiability (SAT) problem (cf. [14): given an instance $S$ of $C$ logical clauses in $V$ boolean variables, is there a truth assignment to the variables such that all clauses are simultaneously satisfied? We will give a number $K$ and construct a convex 3-polytope, of size polynomial in $C$ and $V$ (polynomial size pertains to the binary encoding length), which has a triangulation of size at most $K$ if and only if there is a satisfying truth assignment. In fact we can restrict our discussion to the special case of the SAT problem where each variable appears in three clauses, two of the times negated (see page 259 14]).

Two elementary properties of triangulations will be useful to reach our goal: (1) Every boundary triangular facet $F$ of a polytope is contained in exactly one tetrahedron of a triangulation. The fourth vertex of that tetrahedron is said to triangulate $F$. (2) Simplices of a triangulation cannot intersect in their relative interiors. We will primarily see this behavior in triangles being pierced by an edge of the triangulation, a so-called bad intersection. Our proof combines techniques presented in the articles [6] and [22]:

Consider a long vertex-edge chain on the boundary of a polytope such that the adjacent triangular faces all meet in two points $a$ and $b$ (see Figure 1). The proof of the following lemma can be found in Section 2 of [6]:

Lemma 1.3 Let $P$ be a convex 3-polytope such that the triangles $\left(a, q_{i}, q_{i+1}\right)$ and $\left(b, q_{i}, q_{i+1}\right)$ for $i=0, \ldots, m$ are among its facets, with the additional restriction that conv $\{a, b\} \cap \operatorname{conv}\left\{q_{0}, \ldots, q_{m+1}\right\}=\emptyset$. Let $n$ be the number of vertices of $P$. 
Then, for each triangulation of $P$ that does not use the (interior) edge $(a, b)$ the number of tetrahedra is at least $n+m-3$.

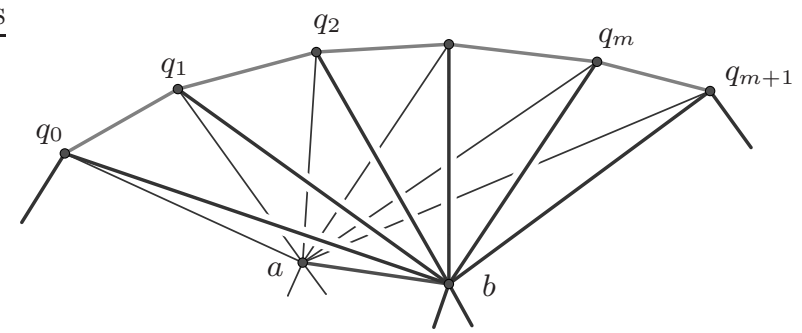

Figure 1: The vertex-edge chain of Lemma 1.3

For us, the number $n+m-3$ will be a relatively large number, such that a triangulation not using $(a, b)$ cannot be small. When using the edge $(a, b)$ we can triangulate $P_{\text {chain }}=\operatorname{conv}\left\{a, b, q_{0}, \ldots, q_{m+1}\right\}$ using the $m+1$ tetrahedra $\left(a, b, q_{i}, q_{i+1}\right)$ for $i=0, \ldots, m$. Call $Q$ the (non-convex) polytope we get after cutting all these tetrahedra out of $P$. Let $n_{Q}$ denote the number of vertices of $Q$. Note that $n=n_{Q}+m$. Suppose the number of tetrahedra in any triangulation of $Q$ (if there is one at all) is bounded above by some number $t$. Then we can bound the size of a minimal triangulation of $P$ using $(a, b)$ by $t+m$. Note that $t$ does not depend on the length $m$ of the vertex-edge chain. Hence, by choosing $m$ large enough (leaving $Q$ as it is) makes

$$
t+m<n_{Q}+2 m-3,
$$

and any close-to-minimal triangulation will have to use $(a, b)$. This argument still holds when we have many vertex-edge chains of the same length $m$ present in other parts of the boundary of the polytope $P$. If $m$ is large enough, a small triangulation is forced to use the edges $(a, b)$ of all these vertex-edge chains.

We also use the famous non-convex Schönhardt polytope [7, 17, 18, 19, 22, 23]. Roughly speaking, a Schönhardt polytope can be obtained by "twisting" the top face of a triangular prism in a clockwise direction (see Figure 2). The three quadrangular sides are then broken up and "bent in", thus creating the nonconvex (reflex) edges $\left(B_{i}, A_{i+1}\right)$ that we call diagonals. The resulting polytope is non-convex and we distinguish two triangular faces the bottom $\left(A_{1}, A_{2}, A_{3}\right)$, and the top or skylight $\left(B_{1}, B_{2}, B_{3}\right)$ not having a reflex edge (Note: whenever dealing with vertices of a Schönhardt polytope, abusing the notation, by an index $i+1$ we mean $(i \bmod 3)+1$. For example $3+1$ gives 1$)$.

The reader can easily verify that Schönhardt polytopes cannot be triangulated using only its six vertices. Imagine the Schönhardt polytope is glued along its bottom face to a bigger polytope. Again, the resulting non-convex polytope can only be triangulated if its top triangle (the skylight) is visible by another vertex (we will rigorously define visible later, but it does correspond to the intuition that every point of the skylight can be joined to the vertex by a straight 


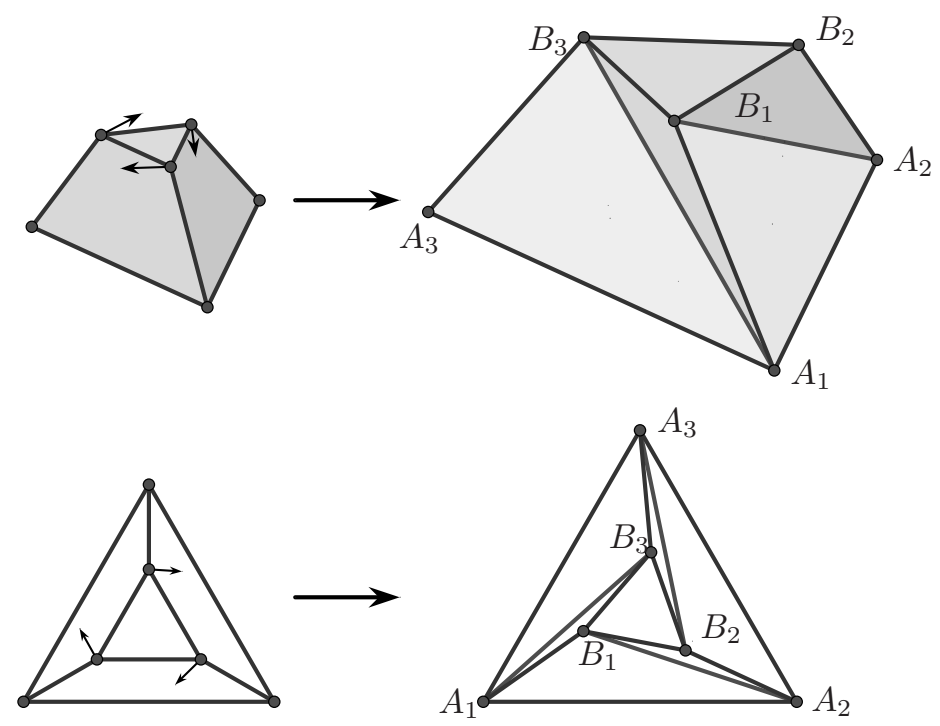

Figure 2: A Schönhardt polytope

line segment. We will show that the triangular cone defined by the hyperplanes containing the faces $\left(B_{i}, B_{i+1}, A_{i+1}\right)$ contains exactly the points that can view the skylight. For this reason we will call this cone the visibility cone.

Now we convexify the Schönhardt polytope by attaching three circular vertexedge chains opposite to the concavities. This will give us a convex polytope that we call the cupola, see Figure 1 . The cupola is usually glued along its bottom face to a bigger convex polytope and obtain a convex polytope $P$. We can combine what we know about vertex-edge chains and about Schönhardt polytopes. Namely, in order to have a small triangulation of $P$, the three diagonals of the Schönhard polytope inside the cupola have to be used. But then, the vertex
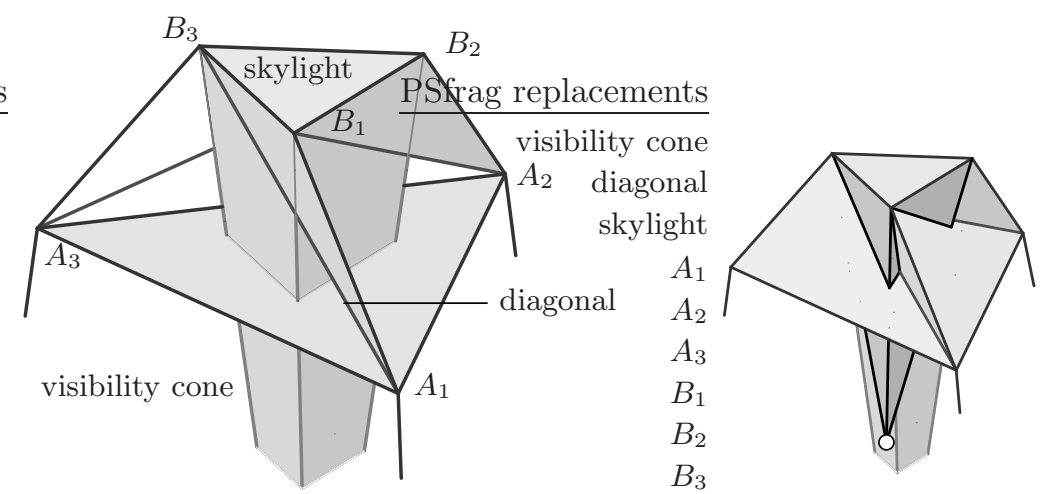

Figure 3: The visibility cone and an invisible vertex 


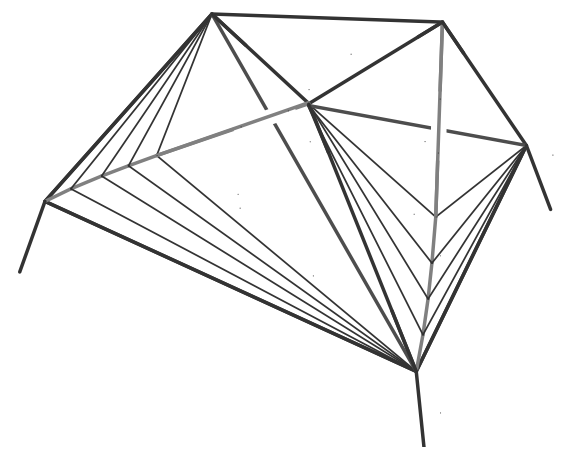

Figure 4: Cupola

triangulating the skylight of the cupola must not be obstructed from seeing the skylight by the diagonals. Hence the vertex has to lie in the visibility cone of the cupola.

In 22 Ruppert and Seidel used SAT to prove that it is NP-complete to decide whether a non-convex polyhedron admits a triangulation. Their constructions used Schönhardt polytopes, and in particular their visibility cones, to do the transformation. In our case, because we need convexity, we glue cupolas, instead of Schönhardt polytopes. They are glued to a bigger frame polytope along their bottom faces. Similar to 22], we have variable cupolas and clause cupolas. The visibility cones of the variable cupolas contain only two truth-setting vertices, one for false and one for true. The visibility cones of the clause cupolas contain as many literal vertices as there are literals in the logical clause. Each variable must choose between a "true" or "false" value. Inside each clause at least one variable will be chosen to be true (to satisfy the clause). We model these logical choices by the geometric choices of which vertex in the visibility cone of a (variable/clause) cupola is used to triangulate the skylight. In addition, our polytope satisfies some blocking conditions: the tetrahedron spanned by the top face of clause cupola and a literal vertex coming from a negated variable $X_{i}$ will improperly intersect the tetrahedron spanned by the top face of the cupola of variable $X_{i}$ and the truth-setting vertex corresponding to true. In this way the choices made for the truth values of the variables and for the literals satisfying the clauses will be consistent. We will call our polytope the logical polytope because it comes from a logical formula.

For the polynomial transformation (from SAT) we need to give an algorithm to compute the coordinates of the logical polytope. The binary encoding length of the polytope, as well as the runtime of the algorithm, have to be polynomial in the encoding length of the SAT instance. Each step of the construction will be polynomial, this is a delicate point in the formalism of our argument. We apply a sequence of these constructions (polynomially many). The coordinates of the vertices of the polytope are potentially singly-exponential, but their binary encoding length is guaranteed to be polynomial. 
Elementary steps of construction include operations such as taking the join of two or three points, intersecting planes and lines, putting points on polynomial curves, etc. The coordinates of the resulting construction elements are therefore polynomials in coordinates of the input elements. On the other hand, we will have requirements on the positions of the points with respect to some planes or other points on lines etc. All these conditions can be formulated as strict polynomial inequalities in coordinates of the construction elements. An essential element of our construction is that our systems of strict polynomial inequalities will depend on one single parameter $\epsilon$. All these polynomial inequalities are satisfied at $\epsilon=0$, but an additional requirement for us is $\epsilon>0$. The following lemma describes a polynomial algorithm to find a number $\epsilon_{0}$ such that all $0<$ $\epsilon \leq \epsilon_{0}$ solve the inequality system.

Lemma 1.4 1. Suppose $p(\epsilon)=a_{d} \epsilon^{d}+\cdots+a_{1} \epsilon+a_{0}$ is a polynomial with $p(0)>0$. Let $\epsilon_{0}(p):=\min \left(1, \frac{a_{0}}{2\left(\left|a_{1}\right|+\cdots+\left|a_{d}\right|\right)}\right)$. Then for $0 \leq \epsilon \leq$ $\epsilon_{0}(p)$ we have $p(\epsilon)>0$.

Hence, the construction of $\epsilon_{0}$ can be done in time polynomial in the encoding length of the coefficients of $p$, and $\epsilon_{0}$ has polynomial encoding length.

2. $p_{1}, \ldots, p_{l}$ are univariate polynomials such that $p_{1}(0)>0, \ldots, p_{l}(0)>0$ then there is a rational number, $\epsilon_{0}>0$, such that $p_{1}(\epsilon)>0, \ldots, p_{l}(\epsilon)>0$ for all $0<\epsilon \leq \epsilon_{0}$. Moreover, the encoding length of $\epsilon_{0}$ is bounded by a polynomial in the encoding length of the coefficients of $p_{1}, \ldots, p_{l}$.

Proof: For $0 \leq \epsilon \leq 1$ we have that $a_{i} \epsilon^{i} \geq-\left|a_{i}\right| \epsilon$. The reason is that for $a_{i} \geq 0$, $a_{i} \epsilon^{i} \geq 0 \geq-\left|a_{i}\right| \epsilon$, and for $a_{i}<0, a_{i} \epsilon^{i}>a_{i} \epsilon=-\left|a_{i}\right| \epsilon$. Hence for $0 \leq \epsilon \leq \epsilon_{0}(p)$

$$
p(\epsilon) \geq \sum_{i=1}^{d}-\left|a_{i}\right| \epsilon+a_{0}>-\sum_{i=1}^{d}\left|a_{i}\right| \frac{a_{0}}{2 \sum_{i=1}^{d}\left|a_{i}\right|}+a_{0}>0 .
$$

For the second part, take the value $\epsilon_{0}\left(p_{1}, \ldots, p_{r}\right):=\min \left(\epsilon_{0}\left(p_{1}\right), \ldots, \epsilon_{0}\left(p_{r}\right)\right)$. Now all the conditions are simultaneously satisfied.

Of course, in general the real solutions of a multivariate system of inequalities coming imposed by geometric requirements may be empty, but our steps of construction reduce everything to sequentially solving easy univariate systems of inequalities.

Here is the organization of our paper. In Section 2 we discuss useful properties of Schönhardt polytopes and of cupolas. Later in the section we explain how to construct and glue cupolas that have a prescribed visibility cone and how to construct visibility cones that fit our purposes. The polynomial transformation to SAT is presented in Section 3. From a given SAT instance we construct a frame polytope to which we then glue the cupolas. In the final Section 14, we discuss the consequences of our result and related complexity problems. As a complement of our main theorem we present a family of polytopes (the socalled stacked polytopes) for which the decision of Theorem 1.1 can be solved in polynomial time. 


\section{Basic Building Blocks}

We recall the notion of beyond a face (see 28]): A point $p$ is beyond a face $F$ of a polytope $P$ if it (strictly) violates all inequalities defining facets of $P$ containing $F$, but it strictly satisfies all other inequalities that define other facets of $P$. The polytope $P_{\text {beyond } F}$ is the (closure of the ) set of all points beyond $F$. We denote by $P \backslash F$ the polyhedron defined by all facet-defining inequalities that do not hold with equality in $F$. This is exactly $P \cup P_{\text {beyond } F}$. In our constructions we will often put one or more points beyond some face, and then take the convex hull. This will only destroy the facets containing this face, and introduce new ones containing the new points. We will say we attach one polytope $P$ to another $Q$ along a facets $F_{P}$ of $P$ and $F_{Q}$ of $Q$ if $P \subseteq Q_{\text {beyond } F_{Q}}$ and $Q \subseteq P_{\text {beyond }} F_{P}$. It is important to observe that the convex hull of their union contains both the face lattices of $P$ and $Q$ without, of course, $F_{P}$ and $F_{Q}$.

\subsection{The Schönhardt Polytope}

Let us turn to a well-known example of a non-convex non-triangulable polytope, the so-called Schönhardt polytope (named after its first occurrence in [23]. See also [19). For the notion of non-convex polytope and what it means to triangulate them we refer to 10 .

Definition 2.1 A Schönhardt polytope (Figure 旬) is a non-convex polytope with six vertices $A_{1}, A_{2}, A_{3}, B_{1}, B_{2}$, and $B_{3}$ and facets $\left(A_{1}, A_{2}, A_{3}\right),\left(B_{1}, B_{2}, B_{3}\right)$, $\left(A_{1}, B_{1}, A_{2}\right), \quad\left(B_{1}, A_{2}, B_{2}\right), \quad\left(A_{2}, B_{2}, A_{3}\right), \quad\left(B_{2}, A_{3}, B_{3}\right), \quad\left(A_{3}, B_{3}, B_{1}\right), \quad$ and $\left(B_{3}, B_{1}, A_{1}\right)$. At exactly the edges $\left(B_{1}, A_{2}\right),\left(B_{2}, A_{3}\right),\left(B_{3}, A_{1}\right)$ the corresponding facets are to span an interior angle greater than $\pi$ (the edges are said to be reflex). These edges are called the diagonals of the Schönhardt polytope. The top face $\left(B_{1}, B_{2}, B_{3}\right)$ is called the skylight of the Schönhardt polytope.

Six points are said to be in Schönhardt position if they are the vertices of a Schönhardt polytope. We say that the skylight is visible from a point $x$ (or $x$ is able to see the skylight, or $x$ is a viewpoint of the skylight) if the tetrahedron spanned by $x$ and the skylight does not intersect any of the diagonals in their relative interior. The visibility cone of the Schönhardt polytope is the triangular cone bounded by the planes $B_{1} B_{2} A_{2}, B_{2} B_{3} A_{3}$, and $B_{3} B_{1} A_{1}$. See Figure 5 .

The use of the word "skylight" is motivated by the idea that the skylight triangle is a glass window and light comes through it illuminating the interior of the Schönhardt polytope defining a cone of light. It is obvious that this non-convex polytope cannot be triangulated (without adding new points): The fourth point of the tetrahedron containing the skylight must be one of $A_{1}, A_{2}$, or $A_{3}$, but the diagonals "obstruct the view" of the skylight from these vertices.

It is our intention to patch the sides of the Schönhardt polytope with vertexedge chains in order to convexify it (and then glue it to a frame polytope). According to Lemma 1.3, a small triangulation of this convex polytope must necessarily contain the diagonals. In this case, the fourth point of the tetrahedron containing the skylight also has to be able to see the skylight. We will show 
where to place the vertex-edge chains in order for them not to be visible from the skylight. Hence, the triangulating vertex has to lie beyond $\left(A_{1}, A_{2}, A_{3}\right)$, we will show that it has to lie in the visibility cone.

Lemma 2.2 Let $A_{1}, A_{2}, A_{3}, B_{1}, B_{2}, B_{3}$ be six points in Schönhardt position. We denote by $C_{A, B}$ the convex hull of the six points. Then

1. All orientations of simplices spanned by four of these six points are determined up to one global sign change. As a consequence, the six points are in convex position, and their convex hull $C_{A, B}$ is an octahedron that has $\left(A_{1}, A_{2}, A_{3}\right)$ and $\left(B_{1}, B_{2}, B_{3}\right)$ as facets and it has edges $\left(A_{i}, B_{i+1}\right)$ $(i=1,2,3)$.

2. There are no points that can see the skylight $\left(B_{1}, B_{2}, B_{3}\right)$ and, at the same time, (1) are beyond either of the edges $\left(A_{i}, B_{i+1}\right)$ of $C_{A, B}$, and (2) are on the side of the plane $B_{1} A_{2} B_{3}$ opposite to $B_{2}$ or similarly for the analogous planes $B_{1} A_{3} B_{2}, B_{2} A_{1} B_{3}$ and the points $B_{3}, B_{1}$ respectively.

3. The visible points beyond the facet $\left(A_{1}, A_{2}, A_{3}\right)$ of $C_{A, B}$ are exactly the points that are also in the visibility cone of the Schönhardt polytope.

In what follows we will use the language of oriented matroids. For the theory of oriented matroids we refer to [9] and [28]. Here we only sketch the necessary definitions and how they are related to the notion of visibility. The orientation of a simplex $\left(x_{1}, x_{2}, x_{3}, x_{4}\right)$, is defined as

$$
\left[x_{1}, x_{2}, x_{3}, x_{4}\right]=\operatorname{sign} \operatorname{det}\left(\begin{array}{cccc}
x_{1} & x_{2} & x_{3} & x_{4} \\
1 & 1 & 1 & 1
\end{array}\right) .
$$

All such orientations make up the chirotope of an oriented matroid (see page 123 in [9]).

Given the oriented matroid of points $x_{1}, \ldots, x_{n}$ in $d$-space, its circuits are functions $C:\left\{x_{1}, \ldots, x_{n}\right\} \mapsto\{+,-, 0\}$ that correspond to so-called minimal Radon partitions. This means that the convex hulls of $C^{+}=\left\{x_{i} \mid C\left(x_{i}\right)=+\right\}$ and $C^{-}=\left\{x_{i} \mid C\left(x_{i}\right)=-\right\}$ intersect in their relative interiors, and $C^{+}$and $C^{-}$ are minimal at that. It is easy to check that the function

$$
C(x)= \begin{cases}(-1)^{i} \cdot[\overbrace{x_{1}, \ldots, x_{d+1}}^{\text {omit } x_{i}}] & , \text { if } x \in\left\{x_{1}, \ldots, x_{d+1}\right\}, \\ 0 & \text { otherwise, }\end{cases}
$$

defines a circuit if it is not identical 0 . In fact, all circuits can be obtained this way. We will compute circuits to use a argument of the following form: $x$ does not see the skylight if and only if there is a circuit such that the positive part is one of the diagonals and negative part is the set containing $x$ and a subset of vertices of the skylight. Since then the tetrahedron spanned by $x$ and the skylight is pierced by the diagonal. 
Important tools to compute simplex orientations are the Grassmann-Plücker relations (see Section 2.4 in [9]): For points $a, b, x_{1}, \ldots, x_{4}$ they state that the set of signs

$$
\left\{\left[a, b, x_{1}, x_{2}\right] \cdot\left[a, b, x_{3}, x_{4}\right],-\left[a, b, x_{1}, x_{3}\right] \cdot\left[a, b, x_{2}, x_{4}\right],\left[a, b, x_{1}, x_{4}\right] \cdot\left[a, b, x_{2}, x_{3}\right]\right\}
$$

is either identical 0 or contains both $\mathrm{a}+$ and $\mathrm{a}-$. The typical use of the Grassmann-Plücker relations is to deduce one orientation when the others are known. We can read the orientations of some of the different tetrahedra from two-dimensional projections (drawings) of the point configurations as in Figure 2. We use a left-handed rule system, i.e. we decide whether the triangle $\left(x_{1}, x_{2}, x_{3}\right)$ is oriented counterclockwise $(+)$ or not $(-)$, also if $x_{4}$ is on our side of the plane spanned by $x_{1}, x_{2}$, and $x_{3}(+)$ or not $(-)$, and multiply these two signs to obtain the orientation $\left[x_{1}, x_{2}, x_{3}, x_{4}\right]$.

Proof of Lemma 2.2:

1. In a Schönhardt polytope, the simplices $\left(A_{1}, A_{2}, A_{3}, B_{1}\right)$, and $\left(A_{1}, A_{2}, A_{3}, B_{2}\right)$ have the same orientation since edges $\left(A_{1}, A_{2}\right)$ and $\left(A_{2}, A_{3}\right)$ are both incident to facet $\left(A_{1}, A_{2}, A_{3}\right)$ and they are both non-reflex edges.

By the above argument, going around the boundary of a Schönhardt polytope, keeping in mind which edges are reflex, we can determine the orientation of 12 simplices up to one global sign change (there are 12 edges). But there are $\left(\begin{array}{l}6 \\ 4\end{array}\right)=15$ simplices formed by the vertices of the Schönhardt polytope. The remaining three simplices are $\left(A_{1}, A_{2}, B_{2}, B_{3}\right),\left(A_{2}, A_{3}, B_{1}, B_{3}\right),\left(A_{1}, A_{3}, B_{1}, B_{2}\right)$. The signs are determined by the following Grassmann-Plücker relations: For $\left(A_{1}, A_{2}, B_{2}, B_{3}\right)$ take $a=A_{1}, b=A_{2}, x_{1}=A_{3}, x_{2}=B_{1}, x_{3}=B_{2}, x_{4}=B_{3}$ (the other two by circular index shift). Then:

$$
\{-\cdot,-\cdot-\cdot+,-\cdot-\} \supseteq\{+,-\}
$$

the equation forces $\left[A_{1}, A_{2}, B_{2}, B_{3}\right]=+$. From the chirotope information it is easy to check that all vertices are in convex position (see description of how to read the facets of the convex hull from the chirotope in Chapter 3 of [9]), and that their convex hull $C_{A, B}$ is indeed an octahedron.

2. We will show that if a point $x$ lies beyond $A_{1} B_{2}$ of $C_{A, B}$, on the side of $B_{1} A_{2} B_{3}$ opposite to $B_{2}$, then $\left(B_{1}, A_{2}\right)$ and the triangle $\left(B_{2}, B_{3}, x\right)$ form a minimal Radon partition in the set of vertices $A_{1}, A_{2}, A_{3}, B_{1}, B_{2}, B_{3}$, and $x$, hence have an interior point in common. This means $x$ cannot see the skylight. For this, we compute the following orientations:

$$
\begin{aligned}
& -\left[B_{1}, B_{2}, B_{3}, x\right]=+, \quad \text { since }\left(B_{1}, B_{2}, B_{3}\right) \text { is a facet of } C_{A, B} \backslash\left(A_{1}, B_{2}\right) \text {, } \\
& +\left[A_{2}, B_{2}, B_{3}, x\right]=+, \quad \text { since }\left(A_{2}, B_{2}, B_{3}\right) \text { is a facet of } C_{A, B} \backslash\left(A_{1}, B_{2}\right) \text {, } \\
& -\left[A_{2}, B_{1}, B_{3}, x\right]=- \text {, from the assumption on } x \text {, } \\
& +\left[A_{2}, B_{1}, B_{2}, x\right]=- \text {, from the Grassmann-Pücker relation below, } \\
& -\left[A_{2}, B_{1}, B_{2}, B_{3}\right]=- \text {, from Part }(1) \text {. }
\end{aligned}
$$


The necessary Grassmann-Plücker relation is the one with $a=B_{1}, b=B_{2}$, $x_{1}=B_{3}, x_{2}=A_{1}, x_{3}=A_{2}$, and $x_{4}=x$ such that

$$
\{-\cdot ?,-\cdot-\cdot-,-\cdot+\} \supseteq\{+,-\}
$$

forces $\left[B_{1}, B_{2}, A_{2}, x\right]=-$.

3. If $x$ is in the visibility cone $V$, then it is, by part (2) of this lemma, on the same side as $B_{3}$ with respect to the plane $B_{1} A_{2} B_{2}$. Hence $A_{2}$ is on opposite side of $B_{3}$ with respect to the plane $B_{1} B_{2} x$. Therefore, the relative interior of the convex hull of $B_{1}$ and $A_{2}$ lies strictly on one side of the plane $B_{1} B_{2} x$, and the tetrahedron $\left(B_{1}, B_{2}, B_{3}, x\right)$ on the other side of this plane. Therefore those two point sets cannot have points in common. By symmetry it follows that the other two diagonals do not obstruct any point of $V$ from seeing the skylight either.

Assume now that a point $x$ is beyond face $\left(A_{1}, A_{2}, A_{3}\right)$, but outside of $V$, i.e. for instance on the $A_{1}$ side of the plane $B_{1} B_{2} A_{2}$. We claim that the pair $\left\{B_{1}, A_{2}\right\},\left\{B_{2}, B_{3}, x\right\}$ forms a circuit in the oriented matroid of the point configuration of the vertices of $C_{A, B}$ and $x$. This means that the triangle $\left(B_{2}, B_{3}, x\right)$ is pierced by the diagonal $\left(B_{1}, A_{2}\right)$ in the relative interior, hence $x$ is not visible.

$$
\begin{aligned}
& -\left[B_{2}, B_{3}, A_{2}, x\right]=-, \text { since }\left(B_{2}, B_{3}, A_{1}\right) \text { is a facet of } C_{A, B} \backslash\left(A_{1}, A_{2}, A_{3}\right), \\
& +\left[B_{1}, B_{3}, A_{2}, x\right]=+, \text { from the Grassmann-Plücker relations below, } \\
& -\left[B_{1}, B_{2}, A_{2}, x\right]=+, \text { from the assumption on } x, \\
& +\left[B_{1}, B_{2}, B_{3}, x\right]=-, \text { since }\left(B_{1}, B_{2}, B_{3}\right) \text { is a facet of } C_{A, B} \backslash\left(A_{1}, A_{2}, A_{3}\right), \\
& -\left[B_{1}, B_{2}, B_{3}, A_{2}\right]=+, \text { from Part }(1) .
\end{aligned}
$$

In this case, we have to apply the Grassmann-Plücker relations twice to get $\left[B_{1}, B_{3}, A_{2}, x\right]=+$. First we deduce $\left[A_{1}, A_{2}, B_{3}, x\right]=-$ from the GrassmannPlücker relation with $a=A_{1}, b=A_{2}, x_{1}=A_{3}, x_{2}=x, x_{3}=B_{2}, x_{4}=B_{3}$ :

$$
\{-\cdot-,-\cdot+\cdot ?,+\cdot+\} \supseteq\{+,-\} \text {. }
$$

Now we use this orientation to formulate $a=A_{2}, b=B_{3}, x_{1}=A_{1}, x_{2}=B_{1}$, $x_{3}=B_{2}, x_{4}=x$ :

$$
\{+\cdot-,-\cdot+\cdot ?,-\cdot+\} \supseteq\{+,-\}
$$

in order to get the desired $\left[A_{2}, B_{3}, B_{1}, x\right]=-$.

\subsection{The Cupola}

Definition 2.3 A polytope $C$ is called a cupola if it has the following properties:

1. the vertices of $C$ are $A_{1}, A_{2}, A_{3}, B_{1}, B_{2}, B_{3}$, and $q_{k}^{i, j}(k=0, \ldots, m+$ $1,(i, j) \in\{(1,2),(2,3),(3,1)\})$, where $q_{0}^{i, j}=A_{i}$ and $q_{m+1}^{i, j}=B_{j}$ are identified. 


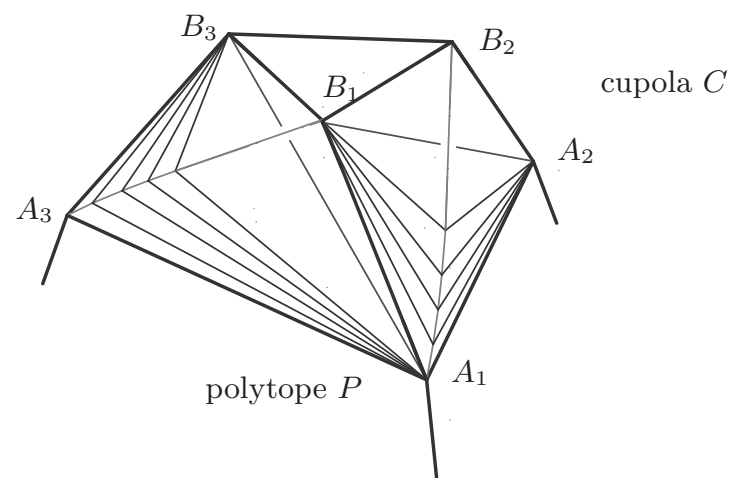

Figure 5: A cupola as part of a larger convex polytope $P$

2. the vertices $A_{1}, A_{2}, A_{3}, B_{1}, B_{2}, B_{3}$ are in Schönhardt position, and $\left(A_{1}, A_{2}, A_{3}\right)$ (the bottom facet) and $\left(B_{1}, B_{2}, B_{3}\right)$ (the skylight) are facets of $C$,

3. the other facets are $\left(B_{i}, q_{k}^{i, j}, q_{k+1}^{i, j}\right)$ and $\left(A_{j}, q_{k}^{i, j}, q_{k+1}^{i, j}\right)$ for $k=0, \ldots, m+1$, $(i, j) \in\{(1,2),(2,3),(3,1)\}$,

4. the vertices $q_{k}^{1,2}(k=1, \ldots, m)$ lie on the side of the plane $B_{1} A_{2} B_{3}$ opposite to $B_{2}$. Similar conditions must hold for $q_{k}^{2,3}$ and $q_{k}^{3,1}$.

Proposition 2.4 Let $C$ be a cupola which is part of a larger polytope $P$, i.e. $Q=P \backslash C$ is a convex polytope and $Q$ and $C$ share the common facet $\left(A_{1}, A_{2}, A_{3}\right)$. Let $n$ be the number of vertices of $P$.

If $T$ is a triangulation of $P$ with the property that the fourth point of the tetrahedron containing the skylight of $C$ is not in the visibility cone of $C$, then there are at least $n+m-3$ tetrahedra in the triangulation.

Proof: If the vertex triangulating the skylight of $C$ is a vertex of on a vertex-edge chain of $C$, then it does not see the skylight by Definition 2.3 (4) and Lemma 2.2 (2). If it is in $Q$ instead, then it has to be beyond the face $\left(A_{1}, A_{2}, A_{3}\right)$ of $C$. Hence by Lemma 2.2 (3) it cannot see the skylight either. Therefore the triangulation $T$ does not use one of the diagonals. By Lemma 1.3 the number of tetrahedra is at least $n+m-3$.

Later, Lemma 1.4 will be used to guarantee that we can place a point beyond a certain face.

\subsection{Constructing a Cupola from a Visibility Cone}

In this subsection we will show that cupolas can be attached to any face of a frame polytope using intermediate polytopes and that the visibility cone can be 
prescribed. The following theorem does not have the full strength we need for the construction. In Section 3, we will use a slightly stronger version which we will present at the end of this section. However, this theorem captures the main ideas used to construct a cupola.

Theorem 2.5 (Cupola Construction from a Given Visibility Cone) Let $F$ be a facet of a 3-polytope $P$, and $V$ be a triangular cone such that $F \cap V$ is a triangle in the relative interior of $F$, and $m$ be a positive integer. Then there is an $m$-cupola $C$ beyond $F$ of $P$ such that $P$ is beyond $\left(A_{1}, A_{2}, A_{3}\right)$ of $C$ and such that $V$ is the visibility cone of $C$. Moreover, the input length of $C$ is polynomial in the input lengths of $P, V$ and $m$.

Before we come to the proof, we will exhibit a necessary condition of the visibility cone $V$ of a cupola $C$ and the facet the cupola is being glued upon. It will imply that we cannot directly attach a cupola to a face (as in [22]), but we have to construct an intermediate polytope first.

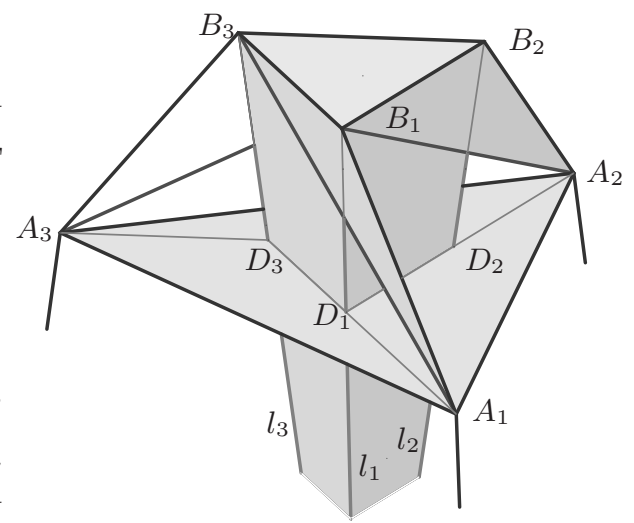

Figure 6: Collinearity condition in the base triangle of a cupola

Lemma 2.6 Let $A_{1}, A_{2}, A_{3}, B_{1}, B_{2}, B_{3}$ be vertices in Schönhardt position. Define $l_{1}$ to be the intersection line of planes $B_{3} B_{1} A_{1}$ and $B_{1} B_{2} A_{2}$, lines $l_{2}$ and $l_{3}$ are defined accordingly (Figure 6 , note that they contain the extreme rays of $V$ ). The lines $l_{1}, l_{2}$, and $l_{3}$ intersect the relative interior of the bottom face $\left(A_{1}, A_{2}, A_{3}\right)$ of a cupola $C$. The intersection points $D_{1}, D_{2}$, and $D_{3}$ are forced to have the following collinearities: $A_{1} D_{1} D_{2}, A_{2} D_{2} D_{3}$, and $A_{3} D_{3} D_{1}$.

Proof: $l_{1}$ enters the Schönhardt polytope $S$ in point $B_{1}$, runs along facet $\left(A_{1}, B_{1}, B_{3}\right)$ until it reaches the edge $\left(A_{1}, B_{3}\right)$ where it goes into the interior of $S$. Then the relative interior of $\left(A_{1}, A_{2}, A_{3}\right)$ contains the point $D_{1}$. In this way, $D_{1}, D_{2}, A_{2}$ are all on the planes $A_{1} A_{2} A_{3}$ and $B_{1} B_{2} A_{2}$.

Proof of Theorem 2.5. We proceed in three steps. The lines $l_{1}, l_{2}, l_{3}$ are defined as in Lemma 6 . 
The bottom triangle $\left(A_{1}, A_{2}, A_{3}\right)$. We will now construct an intermediate polytope beyond $F$ which will have a triangular facet $\left(A_{1}, A_{2}, A_{3}\right)$ which is $(1)$ parallel to $F$, and which is (2) intersected by the cone $V$ in a triangle $\left(D_{1}, D_{2}, D_{3}\right)$ in the relative interior such that (3) the collinearity condition from Lemma 2.6 holds.

To do this, we place a plane $H$ parallel to and slightly above $F$ such that the intersection points $D_{i}$ of $H$ and $l_{i}(i=1,2,3)$. Also $H$ has to be so close to $F$ that the $l_{i}$ do not cross between $H$ and $F$. By prolonging the line segment $D_{3} D_{1}$ slightly beyond $D_{1}$ (staying in $P \backslash F$ ) we obtain point $A_{1}$, analogously construct $A_{2}$ and $A_{3}$ (Figure 7 ). Taking the convex hull of $F$ and the points $A_{1}$, $A_{2}$ and $A_{3}$ gives then the intermediate polytope, whose face $\left(A_{1}, A_{2}, A_{3}\right)$ has the collinearity condition. These constructions are polynomially constructible in the sense of Lemma 1.4.
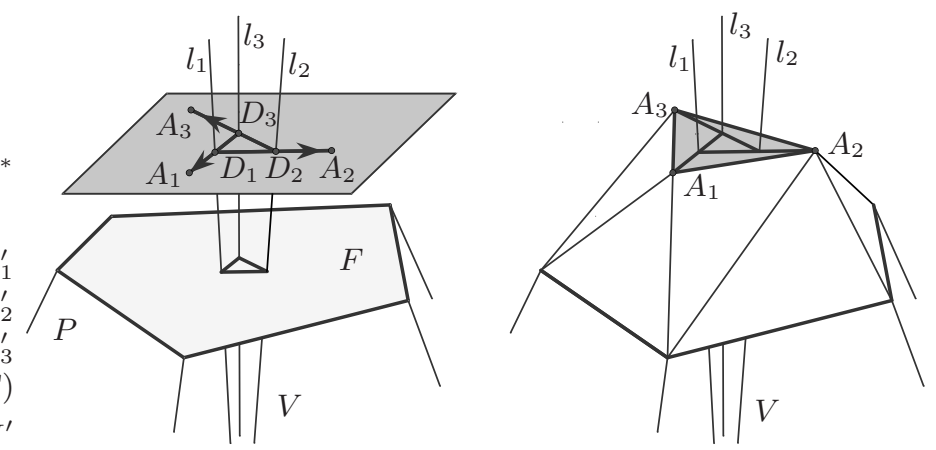

Figure 7: Building the intermediate polytope for the cupola

The frame of the cupola. As in the construction of the bottom facet $\left(A_{1}, A_{2}, A_{3}\right)$, we place a plane $H^{\prime}$ parallel and slightly above this facet. The intersection of $H^{\prime}$ and $V$ is the triangle $\left(B_{1}, B_{2}, B_{3}\right)$ ( $B_{1}$ is on the same extreme ray of $V$ as $D_{1}$ and so on). See figure 8 .

It is clear from the construction that triangles $\left(D_{1}, D_{2}, D_{3}\right),\left(A_{1}, A_{2}, A_{3}\right)$, and $\left(B_{1}, B_{2}, B_{3}\right)$ are parallel and all oriented the same way. Therefore it is not hard to check that the points $A_{1}, A_{2}, A_{3}, B_{1}, B_{2}$, and $B_{3}$ are vertices of a Schönhardt polytope whose visibility cone is $V$. Polynomiality of this part of the construction follows from Lemma 1.4 as well.

Attaching the vertex-edge chains. Now that the frame of a cupola done, i.e. the vertices $A_{1}, \ldots, B_{3}$ in Schönhardt position, it remains to patch the key structures of Lemma 1.3, the vertex-edge chains $q_{i}^{j, k}(i=1, \ldots, m$, $(j, k) \in\{(1,2),(2,3),(3,1)\})$, to the sides of the frame $\operatorname{conv}\left(P \cup\left\{A_{1}, \ldots B_{3}\right\}\right)$.

Given triangular faces $\left(a, q_{0}, q_{m+1}\right)$ and $\left(b, q_{0}, q_{m+1}\right)$ of a convex polytope $P$ and a plane $G$ which (strictly) separates points $q_{0}$ and $q_{m+1}$. We claim that we can construct points $q_{1}, \ldots, q_{m}$ beyond the edge $\left(q_{0}, q_{m+1}\right)$ of $P$ such that the convex hull of $P \cup\left\{q_{i}\right\}$ has the properties of Lemma 1.3 and such that the 

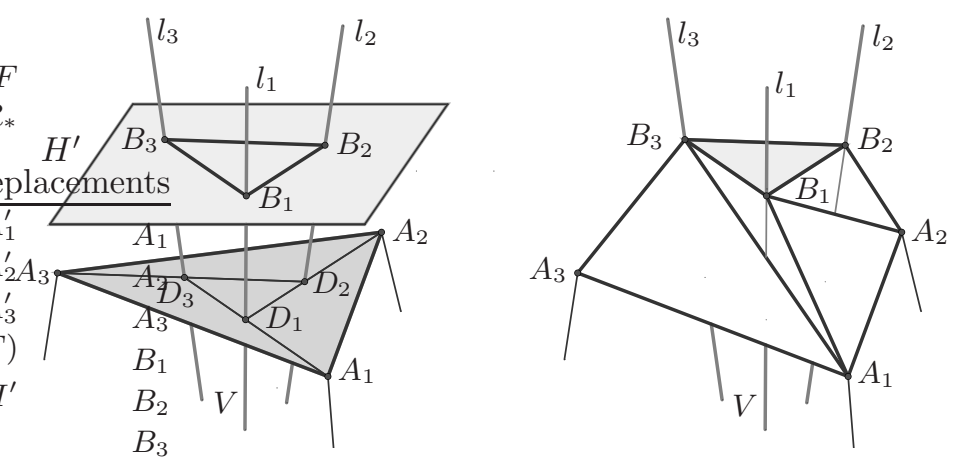

Figure 8: Building the frame of a cupola

points $q_{1}, \ldots, q_{m}$ lie on the same side of $G$ as $q_{0}$. Moreover, the input length of the constructed points is polynomially bounded in the input length of $P$ and $G$.

By applying our claim three times, we will conclude our proof. The vertices $q_{i}^{j, j+1}$ are placed beyond edge $\left(A_{j}, B_{j+1}\right)$, vertices $B_{j}$ and $A_{j+1}$ take the roles of $a$ and $b, G$ is the plane spanned by $B_{j}, A_{j+1}$ and $B_{j+2}$. It is easy to check that this is exactly what we want for Lemma 1.3 and for the cupola conditions.

Now we prove the claim. We will put the points $q_{i}(i=1, \ldots, m)$ on a parabola segment, beyond the edge $\left(q_{0}, q_{m+1}\right)$. Let $H$ be a plane containing $q_{0}$ and $q_{m+1}$ which also intersects the interior of $P$. This plane has the property that it contains points beyond edge $\left(q_{0}, q_{m+1}\right)$. It is constructible in polynomial time. (Let $v$ be the sum of the two normal vectors of planes $a q_{0} q_{m+1}$ and $b q_{0} q_{m+1}$, and $H$ the plane containing $q_{0}$ and $q_{m+1}$ parallel to $v$.)

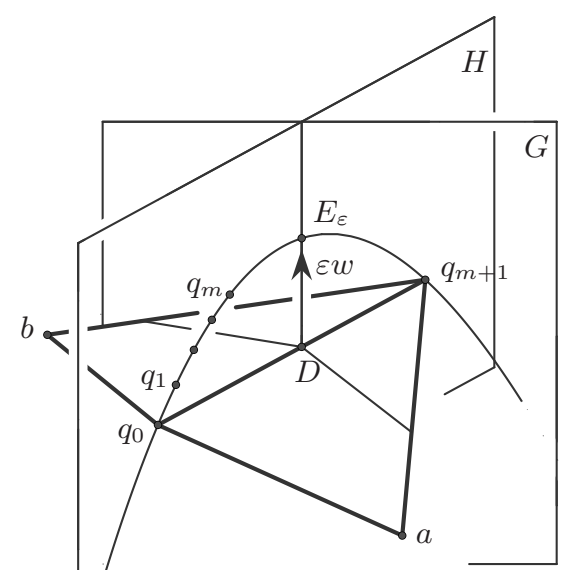

Figure 9: Construction of the vertex-edge chain 
Let now $D$ be the intersection point of $G$ and $\left(q_{0}, q_{m+1}\right)$. Let $w$ be a vector of direction of the intersection line of $G$ and $H$, such that starting at $D$ it is pointing out of $P$. Now let $E_{\varepsilon}=D+\varepsilon w$ for $\varepsilon>0$ to be specified later. For small $\varepsilon, E_{\varepsilon}$ is beyond $\left(q_{0}, q_{m+1}\right)$. Hence the parabola defined according to Lemma 2.7, stated and proved below, by $p(0)=q_{0}, p(1 / 2)=E_{\epsilon}$, and $p(1)=$ $q_{m+1}$ lies entirely in $H$, and for arguments between 0 and 1 passes just beyond $\left(q_{0}, q_{m+1}\right)$. Let $q_{i}=p(i /(4 m))$ for $i=1, \ldots, m$. For small $\varepsilon$ all those points are beyond $\left(q_{0}, q_{m+1}\right)$ and on the same side of $G$ as $q_{0}$ (polynomial conditions, use Lemma 1.4). Also, they are in convex position such that the convex hull of $P \cup\left\{q_{1}, \ldots, q_{m}\right\}$ has exactly the required face lattice.

Lemma 2.7 Let $p_{0}, p_{1}, p_{2}$ three non-collinear points in $\mathbb{R}^{3}$ and $t_{0}, t_{1}, t_{2}$ three distinct real numbers. Then there is a unique curve $p: \mathbb{R} \rightarrow \mathbb{R}^{3}$ such that $p_{0}=p\left(t_{0}\right), p_{1}=p\left(t_{1}\right)$, and $p_{2}=p\left(t_{2}\right)$ which is quadratic in every coordinate. Furthermore, all points on $p(t)$ are in the plane spanned by $p_{0}, p_{1}$, and $p_{2}$, and they are in convex position. Also a plane containing $p(r)$ and $p(l)$ for some $r \neq l$ which does not contain all of $p$ has all points between $l$ and $r$ on one of its sides and all other points on the other side.

Proof: Since $p_{0}, p_{1}, p_{2}$ have to be on the $t_{0}, t_{1}, t_{2}$ positions of the curve

$$
p(t)=\left(\begin{array}{c}
a_{x}+b_{x} t+c_{x} t^{2} \\
a_{y}+b_{y} t+c_{y} t^{2} \\
a_{z}+b_{z} t+c_{z} t^{2}
\end{array}\right),
$$

we have the condition

$$
\left(\begin{array}{ccc}
a_{x} & b_{x} & c_{x} \\
a_{y} & b_{y} & c_{y} \\
a_{z} & b_{z} & c_{z}
\end{array}\right)\left(\begin{array}{ccc}
1 & 1 & 1 \\
t_{0} & t_{1} & t_{2} \\
t_{0}^{2} & t_{1}^{2} & t_{2}^{2}
\end{array}\right)=\left(\begin{array}{ccc}
\vdots & \vdots & \vdots \\
p_{0} & p_{1} & p_{2} \\
\vdots & \vdots & \vdots
\end{array}\right)
$$

By the non-singularity of the Vandermonde matrices, there is a unique solution to $a, b ., c$. given the $p_{i}$ and $t_{i}$.

The curves which are quadratic in every coordinate are linear transforms of the moment curve $m(t)=\left(1, t, t^{2}\right)$. This curve lies entirely in the $x=1$ plane, is convex, and has the condition that it intersected by each plane at most twice (or it is in this plane). All these properties are invariant under linear transformations.

Proposition 2.4 stated that we get a large triangulation if we triangulate the skylight of a cupola by a vertex outside the visibility cone. Now we want to estimate how much smaller a triangulation is if we use a vertex $v$ in the visibility cone instead. We give a relatively small triangulation of the cupola and of the space between the bottom face $\left(A_{1}, A_{2}, A_{3}\right)$ of the cupola and the triangular face $F$ of $P$ with the help of the vertex $v$. 
Proposition 2.8 Let $F$ be triangular face of a polytope $P$, and $C$ an $m$-cupola attached to it according to Lemma 2.5. Let $v$ be a vertex of $P$ in the visibility cone of $C$. Then there is a triangulation of $\operatorname{conv}(\{v\}, F, C)$ with at most $3 m+16$ tetrahedra.

Proof: First of all, we triangulate along the vertex-edge chains using the tetrahedra $\left(B_{i}, A_{i+1}, q_{k}^{i, i+1}, q_{k+1}^{i, i+1}\right)$ for $i=1,2,3$, and $k=0, \ldots, m$.

After removing these tetrahedra, we are left with the union of the Schönhardt polytope on the vertices $A_{1}, A_{2}, A_{3}, B_{1}, B_{2}, B_{3}$, and the convex polytope $\operatorname{conv}\left(\{v\}, F,\left(A_{1}, A_{2}, A_{3}\right)\right)$. This is a non-convex polytope with all edges, except the diagonals, being convex (easy conclusion from Lemma 2.2 and the construction). Since the specified vertex $v$ is inside the visibility cone, it sees all facets of this polytope, except the three facets it is incident to, from the interior. In particular, we can form tetrahedra of all these facets and $v$ and none of them intersect badly. They are 7 tetrahedra for the facets of the Schönhardt polytope (since we do not count the bottom face) and at most 6 for the rest (the convex hull of $F$ and $\left(A_{1}, A_{2}, A_{3}\right)$ has - by a planar graph argument - at most $2 \cdot 6-4=8$ facets, subtracting 2 for $F$ and $\left(A_{1}, A_{2}, A_{3}\right)$ gives 6$)$.

It is this $3 m$ in contrast to the $4 m$ in Proposition 2.4 which makes this triangulation better for large $m$.

\subsection{Constructing a Visibility Cone}

In order to use the cupola as a basic building block, we need to have a visibility cone that contains a specified set of vertices and intersects the relative interior of some face. Once we have that we can construct the cupola as described in the previous section. The set will consist of all vertices lying in a specified plane.

Lemma 2.9 Let $H$ be a plane which intersects the relative interior of some face $F$ of a polytope $P$, and let $S=\left\{v_{1}, \ldots, v_{s}\right\}$ be the set of vertices of $P$ lying in $H$, not including the vertices of $F$. Let $S^{\prime}=\left\{w_{1}, \ldots, w_{s^{\prime}}\right\}$ a set of points in relint $(F) \cap H$. It is possible to construct a triangular cone $V$ which intersects $F$ in a triangle that lies in the relative interior of $F$ and $V$ contains $S$ and $S^{\prime}$ in its interior and no other vertex of $P$.

The reader may not see at this point the purpose of the set $S^{\prime}$, but we will justify it at the end of this section.

Proof: $P \cap H$ is a polygon. Without loss of generality, $F \cap H$ is horizontal and situated on the top of the polygon $P \cap H$ (see Figure 10). Let $l$ be the line connecting the leftmost point of $S^{\prime}$ and leftmost vertex of $S$ (the one encountered first when walking around $P \cap H$ counterclockwise, starting at $F \cap H$ ). Analogously, let $r$ be the line connecting $M$ and the rightmost vertex of $S$.

The area between $l$ and $r$ (in $H$ ) is already a cone containing $S$ and no other vertices of $P$. We will perturb it in a way that the other conditions are satisfied as well. 
First shift $l$ and $r$ parallely outwards, guaranteeing that they still intersect $F \cap H$ in its relative interior (easy open conditions); we obtain $l^{\prime}$ and $r^{\prime}$. Also, let $f^{\prime}$ be a line in $H$ parallel to $F$ just outside $P$, i.e. such that $l^{\prime}$ and $r^{\prime}$ intersect $f^{\prime}$ in the same order as $F \cap H$ (again using Lemma 1.4).
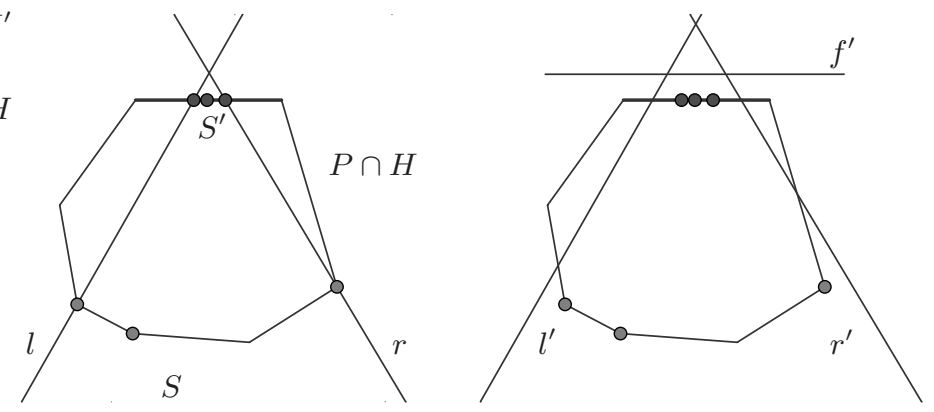

Figure 10: Construction of $l$ and $r$, then $l^{\prime}$ and $r^{\prime}$ (viewed in $H$ )

Now we will rotate $H$ about $l^{\prime}$ and $r^{\prime}$ and $f^{\prime}$, getting three planes bounding the desired triangular cone: Let $H$ be oriented in some way, and $a_{H} x \geq b_{H}$ be its defining inequality. Let $v$ be some point which lies on the positive side of $H$. Let $G_{l^{\prime}}$ be the plane through $l^{\prime}$ and $v$. By construction, all vertices in $S$ lie on the same side of $G_{l^{\prime}}$, so we can orient it such that $S$ is on its positive side. Let $a_{l^{\prime}} x \geq b_{l^{\prime}}$ be its defining inequality. Perform the same construction for $r^{\prime}$ and $f^{\prime}$ obtaining $G_{r^{\prime}}$ and $G_{f^{\prime}}$, also orienting them in a way that $v$ is on their respective positive sides. Let $G_{l^{\prime}}^{\varepsilon}$ be the plane defined by $\left(a_{H}+\varepsilon a_{l^{\prime}}\right) x \geq b_{H}+\varepsilon b_{l^{\prime}}$. This plane contains $l^{\prime}$ and for small $\varepsilon$ it is very close to $H$. Hence, it is the rotation of $H$ about $l^{\prime}$ in the direction of plane $G_{l^{\prime}}$. Also let $G_{r^{\prime}}^{\varepsilon}$ be defined by $\left(a_{H}+\varepsilon a_{r^{\prime}}\right) x \geq b_{H}+\varepsilon b_{r^{\prime}}$, and $G_{f^{\prime}}^{\varepsilon}$ be defined by $\left(-a_{H}+\varepsilon a_{f^{\prime}}\right) x \geq-b_{H}+\varepsilon b_{f^{\prime}}$.

Obviously, all points in $S$ and in $S^{\prime}$ are on the positive sides of the planes $G_{l^{\prime}}^{\varepsilon}, G_{r^{\prime}}^{\varepsilon}$, and $G_{f^{\prime}}^{\varepsilon}$. For small $\varepsilon>0$, these planes do not "sweep" over vertices of $P$ which are not in $S$, and it is easy to see that in this case, there are no vertices of $P$ that satisfy all three new inequalities. Also for small $\varepsilon$, the points in $F$ satisfying all three inequalities define a triangle in the relative interior of $F$ with endpoints $G_{l^{\prime}}^{\varepsilon} \cap G_{r^{\prime}}^{\varepsilon} \cap F, G_{r^{\prime}}^{\varepsilon} \cap G_{f^{\prime}}^{\varepsilon} \cap F$, and $G_{f^{\prime}}^{\varepsilon} \cap G_{l^{\prime}}^{\varepsilon} \cap F$. Hence, the set of all points satisfying the three inequalities is a triangular cone $V$ with the desired properties. The conditions on $\varepsilon$ are open polynomial conditions according to Lemma 1.4.

This lemma can be used to build one cupola over the facet $F$. However, there might be problems if we keep on constructing around the polytope, like adding more cupolas over other facets of $P$. The visibility cone we just constructed might "catch" points we construct later. But these constructions all happen beyond facets of $P$, so we can use the following lemma to construct all cupolas one after the other without their visibility cones catching extra vertices.

Lemma 2.10 Let $H_{1}, \ldots, H_{n}$ hyperplanes, intersecting facets $F_{1}, \ldots, F_{n}$ of a 


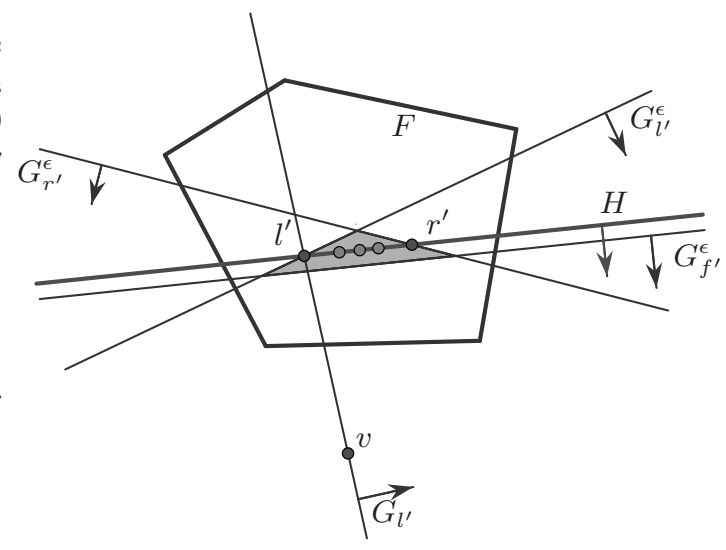

Figure 11: Rotated hyperplanes, viewed by their intersections with $F$

polytope $P$ with the restriction that $F_{i} \cap H_{j}=\emptyset$ for all $i \neq j$. Then $P_{\text {beyond } F_{i}} \cap$ $H_{j}=\emptyset$ for all $i \neq j$.

Proof: Assume there is a point $u$ in $P_{\text {beyond }} F_{i} \cap H_{j}(i \neq j)$. Then this point also lies in $\left(P \backslash F_{i}\right) \cap H_{j}$, but on the non-positive side of $F_{i}$. Let $v$ be a point in $F_{j} \cap H_{j}$, Then $v$ is also in $\left(P \backslash F_{i}\right) \cap H_{j}$ (since $\left.F_{j} \subseteq P \subseteq P \backslash F_{i}\right)$, but on the positive side of $F_{i}$. Hence, there must be a point $w$ on the line segment $[u, v]$ which is on the hyperplane containing $F_{i}$. The whole segment lies in $P \backslash F_{i}$, hence every point on it has to satisfy all of $P$ 's defining inequalities except that of $F_{i}$. So $w$ lies in the facet $F_{i}$. But it also lies in $H_{j}$ (the whole line segment does), which contradicts the assumption $F_{i} \cap H_{j}=\emptyset$.

In Section 3.1 we will need an additional condition: Given a set of lines in the plane $H$ (of Lemma 2.9) that pierce the face $F$, we want to be sure that these lines also pierce the skylight of the constructed cupola. (This condition will play an important role when we want to force so-called blocking conditions, see Section 3: At some point two tetrahedron spanned by two skylights and two respective visible vertices $v$ and $v^{\prime}$ will have to intersect in their interiors. This is already guaranteed if the corresponding lines $g$ and $g^{\prime}$ intersect inside the polytope.)

The next theorem specifies the way in which we will use all the preceding lemmas in our construction in Section 3:

Theorem 2.11 (Full-Strength Cupola Construction) Let $H_{i}$ be planes that intersect facets $F_{i}$ of a polytope $P$ in their relative interiors such that $F_{i} \cap H_{j}=\emptyset$ for all $i \neq j$. Let $S_{i}=\left\{v_{1}^{i}, \ldots, v_{s_{i}}^{i}\right\}:=\left(\operatorname{vert}(P) \cap H_{i}\right) \backslash F_{i}$, and $L_{i}=\left\{g_{1}^{i}, \ldots, g_{s_{i}^{\prime}}^{i}\right\}$ sets of lines. Assume further that each of the lines $g_{j}^{i}$ pierces the relative interior of $F_{i}$ and is incident to some $v_{k}^{i}$.

Then we can sequentially construct all cupolas $C_{i}$ beyond the faces $F_{i}$ such that in the resulting polytope their visibility cones contain $S_{i}$ and no other ver- 
tices. In addition, the skylight of the cupola over each $F_{i}$ is pierced by the lines in $L_{i}$.

Proof: The theorem follows from the ideas in Lemmas 2.5 and 2.9. In the construction of the visibility cone over facet $F_{i}$, we invoke Lemma 2.9 with the polytope $P \cup \bigcup_{j \neq i} P_{\text {beyond }} F_{j}$. The set $S_{i}^{\prime}$ is of course $\left\{l \cap F_{i} \mid l \in L_{i}\right\}$. The cupola construction was such that the cupolas over $F_{j}$ were always beyond the facet $F_{j}$, so the constructed visibility cones contain no vertices of the other cupolas. In order to have the lines in $L_{i}$ pierce the skylight of cupola $i$ we have to alter the construction of the cupola in Lemma 2.5: when we put the planes parallel to $F$, we do it in such a way that the triangles $\left(A_{1}, A_{2}, A_{3}\right)$ and then $\left(B_{1}, B_{2}, B_{3}\right)$ are pierced by these lines. These are both open conditions on the distance of the planes to $F$.

\section{The Transformation from SAT}

It is our intention to model the well-known satisfiability problem (SAT) using the visibility cones of cupola polytopes. Just as Ruppert and Seidel did in [22], we will restrict our attention to special SAT instances: each variable appears exactly three times, twice unnegated and once negated. This is not really necessary, but simplifies explanations. For our purpose this restriction is appropriate because the SAT problem remains NP-complete even for instances where each variable or its negation appear at most three times (see references on page 259 in [14]). In addition, note that a change of variables can be used to change a non-negated variable into a negated variable if necessary. Also note that if a variable appears only negated or only positive the variable and the clauses that contain it can be discarded. Finally, if a variable appears exactly once positive and exactly once negated then it can be eliminated by combining the two clauses that contain the two variables into one. From now on, all logical formulas will have the properties that each variable occurs exactly two times unnegated and exactly once negated. The formula

$f=\left(X_{1} \vee \neg X_{2} \vee X_{3} \vee \neg X_{4}\right) \wedge\left(\neg X_{1} \vee X_{2} \vee \neg X_{3} \vee X_{4}\right) \wedge\left(X_{1} \vee X_{2} \vee X_{3} \vee X_{4}\right)$

is such a special SAT formula. The figures in this section will correspond to this particular instance.

In Section 3.1, we will define the logical polytope associated to a given logical formula. In Section 3.2 we will compute a number $K$ and see that the logical polytope has a triangulation with $\leq K$ tetrahedra if and only if the logical formula is satisfiable. Finally, in Section 3.3 we will give an algorithm to construct explicit coordinates of a logical polytope.

\subsection{The Logical Polytope}

In the logical polytope there will be a cupola for each clause and one for each variable and its negation. The cupolas will be glued to a frame polytope which 
resembles a wedge. Look carefully at Figure 12 for an example of the overall structure.

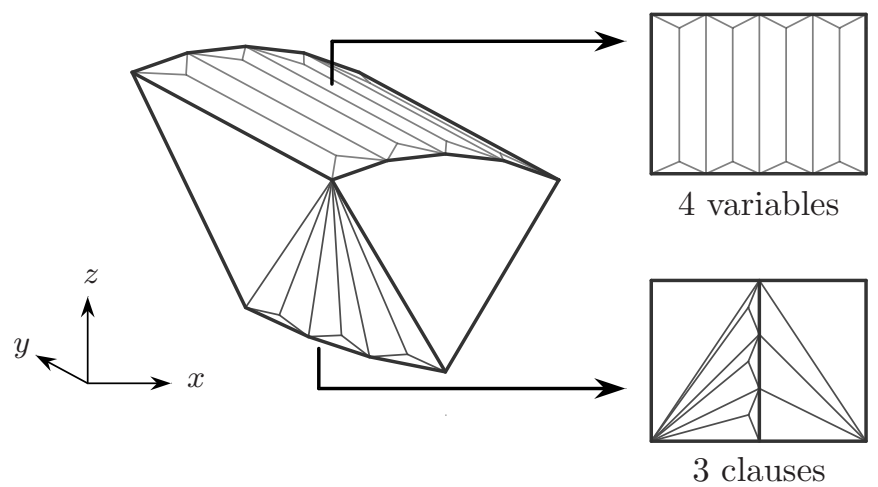

Figure 12: Sketch of the logical polytope

Figure 13 displays the view of the lower hull of the frame polytope, seen from the inside, i.e. from above. The sharp part of the wedge consists of $2 C+1$ vertices (where $C$ is the number of clauses) $c_{0}, \ldots, c_{2 C}$. We call this part of the frame polytope the spine. We attach the clause cupola associated with clause $i$ to the triangle $\left(c_{2 i}, c_{2 i+1}, c_{2 i+2}\right)$ (shaded in the picture).
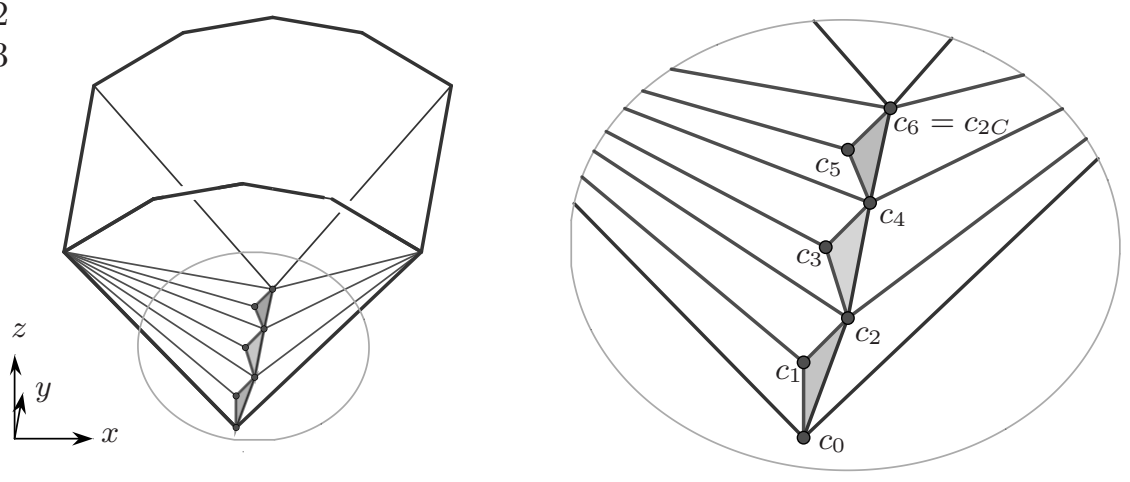

Figure 13: The spine of the wedge: here the clause cupolas are attached

On top of this wedge structure we will put a series of roofs. They are triangular prisms, spanned by the two triangles $\left(x_{T}^{i}, x_{F}^{i}, z_{A}^{i}\right)$ and $\left(x_{L}^{i}, z_{R}^{i}, z_{B}^{i}\right)$, one for every variable $X_{i}$ of the logical formula. The variable cupolas will be attached to the triangular facet $\left(z_{L}^{i}, z_{R}^{i}, z_{B}^{i}\right)$, the back gables (the triangular faces are shaded in Figure 14).

The variable cupola of variable $X_{i}$ is such that its visibility cone contains exactly the front vertices vertices $z_{T}^{i}$ and $z_{F}^{i}$. We will use these cupolas to read from a small triangulation of the polytope the logical value of variables 


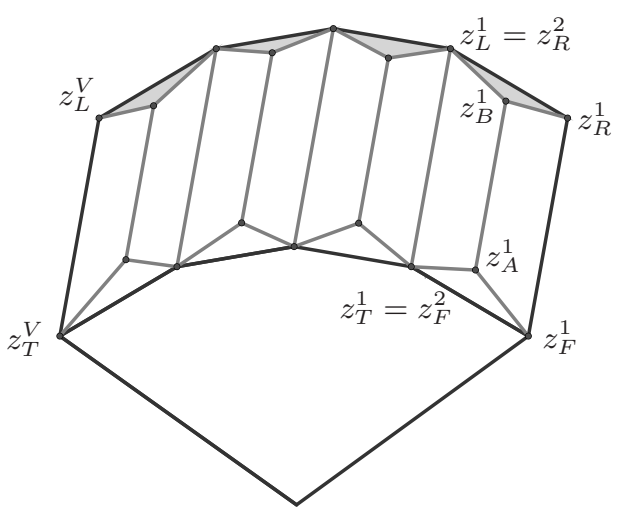

Figure 14: The roofs, back gables shaded

according with the following rule: if the truth-setting vertex $z_{T}^{i}$ associated to the $i$ th logical variable is used to triangulate the skylight of the cupola for variable $i$, then we set $X_{i}=$ true. If the truth-setting vertex used to triangulate the skylight of the cupola for variable $i$ is instead $z_{F}^{i}$ then $X_{i}=$ false.

Beyond the quadrilateral face containing $z_{T}^{i}$ we will place the literal vertices $x_{1}^{i}$ and $x_{2}^{i}$ which corresponds to the positive occurrences of $X_{i}$ in the logical formula. Beyond the other quadrilateral face we will place the other literal vertex $\overline{x_{3}^{i}}$ which correspond to the negated occurrence of $X_{i}$. These vertices are in the visibility cones of the three cupolas of the clause where variable $X_{i}$ or its negation appears.

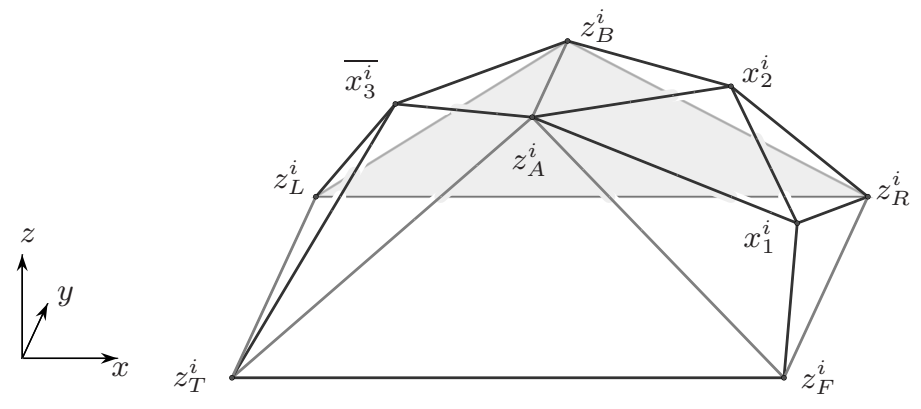

Figure 15: A roof, back gable shaded, $z$-coordinate superelevated

We list the five conditions on the logical polytope which are necessary for the transformation to work in both ways, i.e. a small triangulation yields a satisfying truth assignment for our logical formula and vice versa.

Definition 3.1 For a given logical formula, a logical polytope is a threedimensional polytope $P$ that satisfies the following conditions: 
1. (Convexity) The logical polytope must be convex and the face lattice is as we just described it.

2. (Visibility) The literal vertices $x_{1}^{i}, x_{2}^{i}$, and $\overline{x_{3}^{i}}$ are vertices in the visibility cone associated to their respective clause cupolas, but of no other clause visibility cone. The vertices $z_{T}^{i}, z_{F}^{i}$ are the only vertices in the visibility cones of the ith variable cupola.

3. (Blocking) This constraint ensures that the assignment of true or false values for variables is done consistently, i.e. the positive (negative) literals can be used to make their clauses true if and only if the variable is set true (false).

Concretely, the tetrahedron spanned by $z_{F}^{i}$ and the skylight of the cupola of variable $X_{i}$ intersects the interior of tetrahedron spanned by $x_{1}^{i}$ (by $x_{2}^{i}$ ) and the skylight of the clause cupola corresponding to $x_{1}^{i}$ (to $x_{2}^{i}$ ). Also the tetrahedron spanned by $z_{T}^{i}$ and the skylight of the cupola of variable $X_{i}$ intersects the interior of the tetrahedron spanned by $\overline{x_{3}^{i}}$ and the skylight of the clause cupola corresponding to it. See Figure 10 for an example.

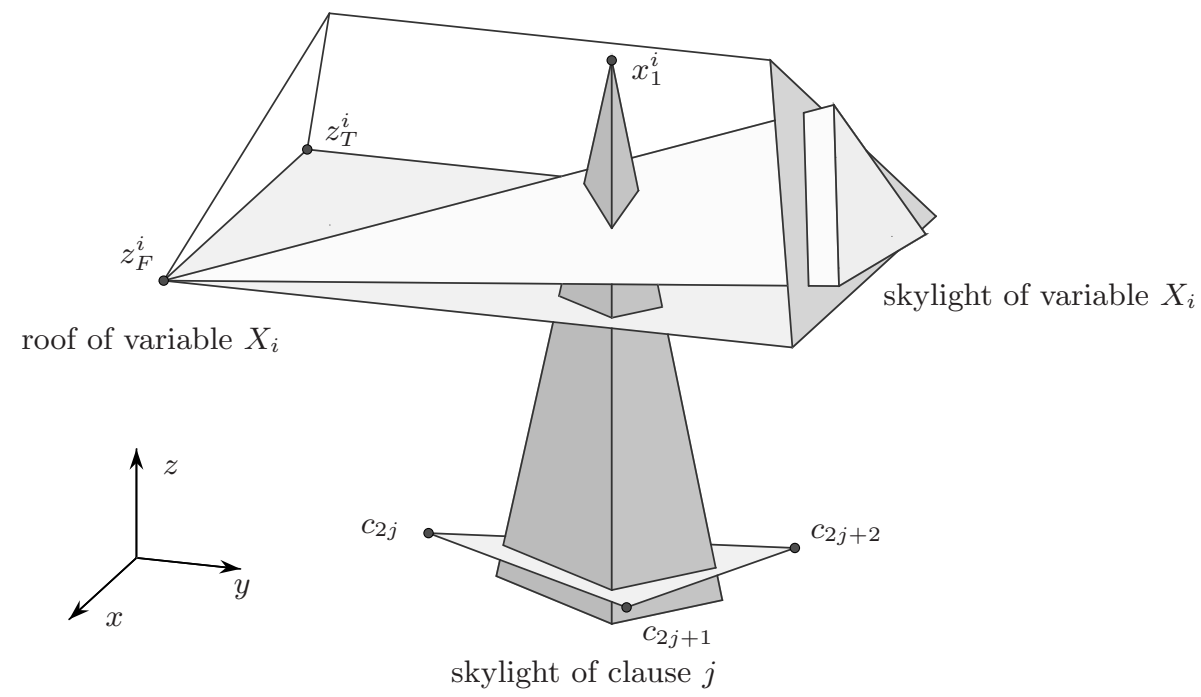

Figure 16: Blocking for consistent logical values

4. (Non-blocking) Using the vertex $z_{T}^{i}$ to triangulate the interior of the $i$-th variable cupola should not prevent the non-negated literal vertices from seeing their associated cupolas. Concretely, if $j$ is the clause corresponding to the literal vertex $x_{1}^{i}$, then tetrahedra $\left(z_{T}^{i}, z_{L}^{i}, z_{R}^{i}, z_{B}^{i}\right)$ and $\left(x_{1}^{i}, c_{2 j-2}, c_{2 j-1}, c_{2 j}\right)$ do not intersect at all. The canonical analogue shall hold for $x_{2}^{i}$ and $\overline{x_{3}^{i}}$ (for $\overline{x_{3}^{i}}$ replace $z_{T}$ by $z_{F}$ ). 
5. (Sweeping) We follow the same sweeping procedure proposed by Ruppert and Seidel [2] we will need that

(a) the variable $x_{1}^{i}$ is to the "left" (negative $x$ direction) of the planes $c_{2 k-1} c_{2 k} z_{F}^{i}, c_{2 k} c_{2 k+1} z_{F}^{i}$, and $c_{2 k-1} c_{2 k+1} z_{F}^{i}$ for $0 \leq k \leq C-1$.

(b) $x_{2}^{i}$ is to the "left" of the planes $c_{2 k-1} c_{2 k} x_{1}^{i}, c_{2 k} c_{2 k+1} x_{1}^{i}$, and $c_{2 k-1} c_{2 k+1} x_{1}^{i}$ for $0 \leq k \leq C-1$.

(c) $\overline{x_{3}^{i}}$ is to the "left" of the planes $c_{2 k-1} c_{2 k} z_{F}^{i}, \quad c_{2 k} c_{2 k+1} z_{F}^{i}$, and $c_{2 k-1} c_{2 k+1} z_{F}^{i}$ for $0 \leq k \leq C-1$.

(d) $z_{T}^{i}$ is to the "left" of the planes $c_{2 k-1} c_{2 k} x_{2}^{i}, c_{2 k} c_{2 k+1} x_{2}^{i}, c_{2 k-1} c_{2 k+1} x_{2}^{i}$, $c_{2 k-1} c_{2 k} \overline{x_{3}^{i}}, c_{2 k} c_{2 k+1} \overline{x_{3}^{i}}$, and $c_{2 k-1} c_{2 k+1} \overline{x_{3}^{i}}$ for $0 \leq k \leq C-1$.

\subsection{Using the Logical Polytope}

Lemma 3.2 Let $P$ be the Logical polytope, $m$ the number of vertices on each vertex-edge chain, $n$ be the total number of its vertices. For a SAT formula containing $C$ clauses on $V$ vertices there are polynomials $K(C, V)$ and $m(C, V)$ such that a logical polytope with $m=m(C, V)$ vertices on each vertex-edge chain admits a triangulation with $\leq K=K(C, V)=n+m-4$ tetrahedra if and only if there is a satisfying truth assignment to the variables of the logical formula.

Proof: If a triangulation $T$ of the polytope has $\leq n+m-4$ tetrahedra, then by Proposition 2.4 the skylight of each cupola is triangulated by a vertex in the visibility cone of the cupola. In particular, one of $z_{F}^{i}$ and $z_{T}^{i}$ is chosen to triangulate the cupola corresponding to variable $X_{i}$ for each $i$. We claim that assigning to $X_{i}$ the truth value according to this choice $\left(z_{F} \mapsto\right.$ false, $z_{T} \mapsto$ true $)$ satisfies all clauses of the formula.

Each clause cupola skylight is triangulated by one of the literal vertices, say clause $j$ by the positive literal vertex $x_{1}^{i}$ ( or $x_{2}^{i}$ ). By the blocking conditions, it cannot be the case that the variable skylight of $X_{i}$ is triangulated by $z_{F}^{i}$ since these tetrahedra would intersect badly. So we had set $X_{i}$ to true. Having $x_{1}^{i}$ ( or $x_{2}^{i}$ ) in clause $j$ 's visibility cone meant that variable $X_{i}$ appears unnegated in this clause. If the skylight of clause cupola $j$ is triangulated by $\overline{x_{3}^{i}}$, by the same argument $X_{i}$ was set to false, and clause $j$ satisfied by the literal $\neg X_{i}$. Hence all clauses are satisfied.

Now we need to prove the converse. If there is a true-false assignment that satisfies all logical clauses we must find a triangulation that has no more than $K$ tetrahedra. For that we construct a "small" triangulation. There are three different kinds of tetrahedra: the ones triangulating the cupolas, the ones triangulating the roofs, and the ones triangulating of the rest of the wedge. We know how to triangulate a cupola if we know a vertex in its visibility cone (see the proof of Prop. 2.8). For the rest we will now follow a sweeping procedure which was first described by Ruppert and Seidel 22].

The sweeping triangulation proceeds by triangulating "slices" that correspond to the different variables $X_{1}$ to $X_{V}$, i.e. from right to left. The variable 
roofs are arranged sequentially for exactly this purpose. A slice is roughly speaking the part of the tetrahedra between a roof and vertices of the spine. After the $i$ th step of the process the partial triangulation will have triangulated the first $i$ slices. The part of the boundary of the partial triangulation that is inside the logical polytope will form a triangulated disk. We will call it the interface following the convention of Ruppert and Seidel. It contains the following triangles:

$\left(z_{T}^{i}, c_{2 C}, z_{L}^{i}\right)$ and $\begin{cases}\left(z_{T}^{i}, c_{2 j-2}, c_{2 j}\right) & : \text { if clause } j \text { is satisfied by one of } \\ \left(z_{T}^{i}, c_{2 j-2}, c_{2 j-1}\right) & \text { the first } i \text { variables, or } \\ \text { and }\left(z_{T}^{i}, c_{2 j-1}, c_{2 j}\right) \quad: & \text { otherwise, }\end{cases}$

for all $j=1, \ldots, C$. Before the first step, the partial triangulation is empty.

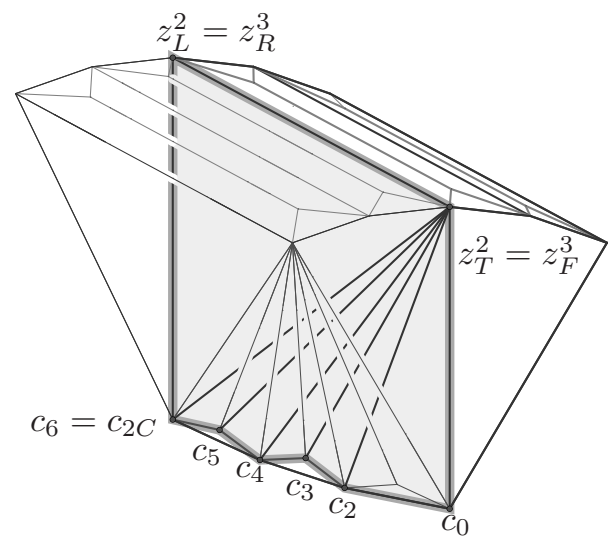

Figure 17: The interface after step 2

After the last step the partial triangulation will cover the whole logical polytope. In general, the vertices of the $i$ th roof will see all triangles of the interface and will be used as apexes to form new tetrahedra to add to the current partial triangulation. This way the interface will slowly move from right to left.

Now we describe in detail the triangulation step for the $i$ th variable $X_{i}$. Since we are only concerned with roof vertices in roof $i$, we will drop all superscripts. The triangulation step depends on whether $X_{i}$ is set true or false in the satisfying assignment. Let us consider first the case $X_{i}=$ true:

The point $z_{T}$ is used to triangulate the interior of the variable cupola associated to $X_{i}$ according to Prop. 2.8. From $z_{T}$ we also form six tetrahedra with the following triangles: $\left(z_{L}, \overline{x_{3}}, z_{B}\right),\left(\overline{x_{3}}, z_{B}, z_{A}\right),\left(z_{B}, z_{A}, x_{2}\right),\left(z_{B}, x_{2}, z_{R}\right)$, $\left(z_{A}, x_{1}, x_{2}\right)$, and $\left(x_{1}, z_{A}, z_{F}\right)$.

Now we come to the part of the triangulation which gave the sweeping procedure its name. We form the tetrahedra between $x_{1}$ and the current interface triangles. This is possible by part (a) of condition 5 . We also use the tetrahedron $\left(x_{1}, z_{T}, c_{0}, z_{F}\right)$. This is illustrated in the transition from a. to b. in Figure 19. The interface advances to $x_{1}$, i.e. if $\left(z_{F}, c_{j}, c_{k}\right)$ was an interface triangle 

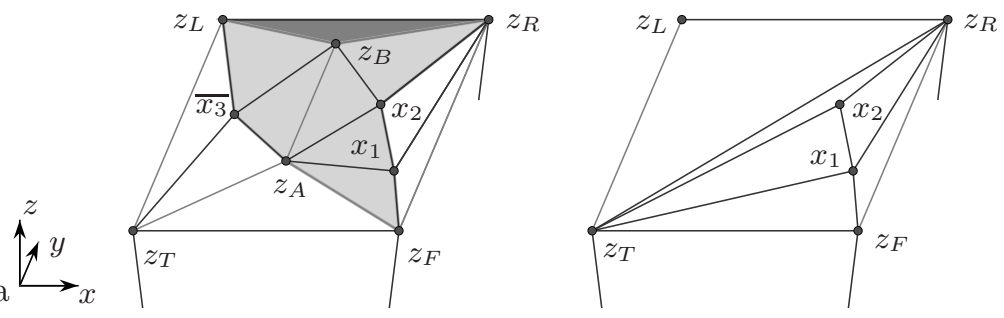

Figure 18: Removing the tetrahedra spanned by $z_{T}$ and the shaded triangles
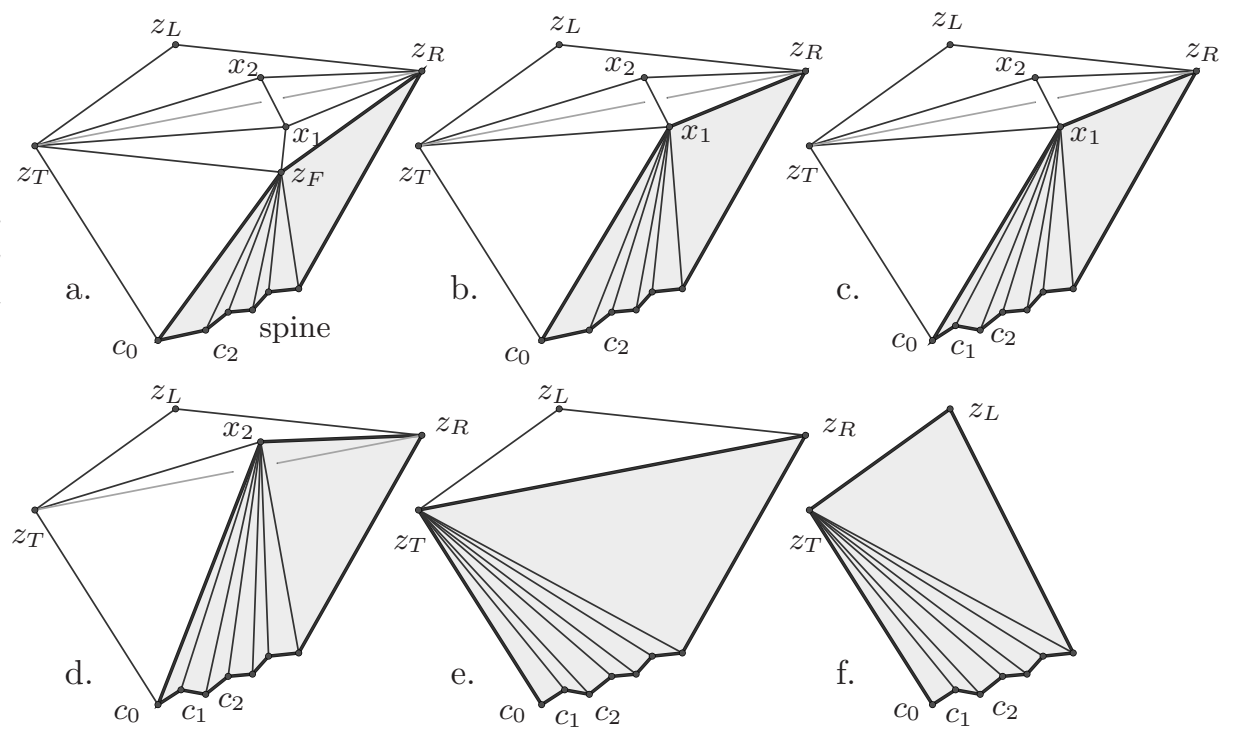

Figure 19: The sweep

before, now $\left(x_{1}, c_{j}, c_{k}\right)$ is an interface triangle. Also $\left(z_{F}, c_{2 C}, z_{R}\right)$ is replaced by the triangle $\left(x_{1}, c_{2 C}, z_{R}\right)$.

Since $X_{i}$ is set to true we can use $x_{1}$ to triangulate its clause cupola according to Prop. 2.8. We only do this if the clause cupola has not been previously triangulated using an other literal vertex. The condition 2 ensures that $x_{1}$ is in the visibility cone of the clause cupola coming from the clause that contains the unnegated literal $X_{i}$. Furthermore, condition 4 ensures that we can actually perform this triangulation of the clause cupola without badly intersecting the tetrahedra of the variable cupola. In Figure 19.c. we see that if $x_{1}$ is used to triangule clause $j$ 's cupola, then the interface triangle $\left(x_{1}, c_{2 j-2}, c_{2 j}\right)$ is replaced by the two triangles $\left(x_{1}, c_{2 j-2}, c_{2 j-1}\right)$ and $\left(x_{1}, c_{2 j-1}, c_{2 j}\right)$.

We repeat this procedure with $x_{2}$, i.e. form tetrahedra with $x_{2}$ and the current interface triangles, and then use $x_{2}$ to triangulate its clause cupola if necessary (Figure 19. d.). This is possible by part (b) of condition 5. We continue 
by forming tetrahedra using $z_{T}$ as apex (Figure 19. e, possible by condition 5 , part (d)). At last, we will include the triangle $\left(c_{2 C}, z_{L}, z_{B}\right)$. All these triangles are visible by part (d) of the sixth condition. After all these tetrahedra are added the interface is ready for the next variable.

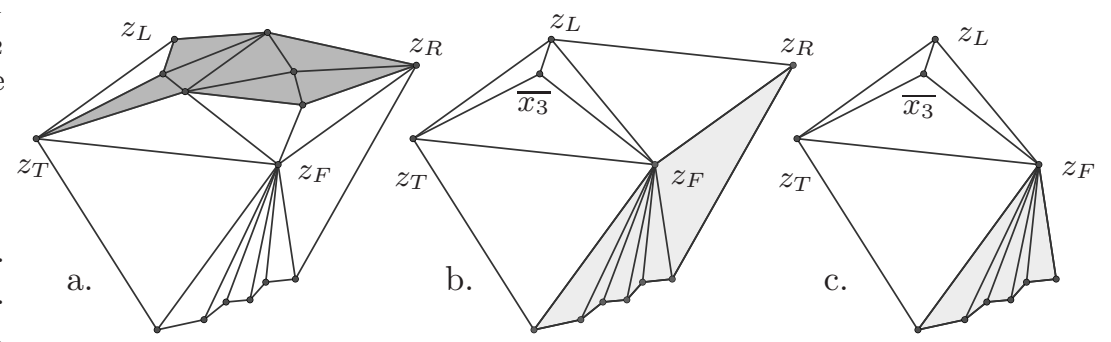

Figure 20: The sweep for $X_{i}=$ false

Let us now consider the triangulation step in the case $X_{i}$ is set to be false: We use the vertex $z_{F}$ to triangulate the variable cupola as well as seven faces of the roof (see Figure 20): $\left(z_{T}, \overline{x_{3}}, z_{A}\right),\left(\overline{x_{3}}, z_{A}, z_{B}\right),\left(\overline{x_{3}}, z_{L}, z_{B}\right),\left(z_{B}, z_{A}, x_{2}\right)$, $\left(z_{B}, x_{2}, z_{R}\right),\left(z_{A}, x_{2}, x_{1}\right),\left(x_{2}, x_{1}, z_{R}\right)$. The reader can see that on the roof we are leaving only the vertex $\overline{x_{3}}$. Next the tetrahedron $\left(z_{F}, z_{L}, z_{R}, c_{2 C}\right)$ is cut out. Hereby the interface triangle $\left(z_{F}, z_{R}, c_{2 C}\right)$ is replaced by $\left(z_{F}, z_{L}, c_{2 C}\right)$ (Figure 20.c.). Then $\overline{x_{3}}$ will be used as apex with the triangles in the interface. If the negated literal $\overline{X_{i}}$ is used to satisfy its clause $j$, the $j$ th clause cupola is triangulated by $\overline{x_{3}}$. The interface advances as in the true-case. Then $z_{T}$ can be used to form tetrahedra with the triangles in the interface. In the end the interface is again ready for the next variable.

How may tetrahedra can such a triangulation have? Triangulating all cupolas with a vertex in their visibility cones yields at most $(3 m+16)(C+V)$ tetrahedra (Prop. 2.8). In one step of the sweeping triangulation the tops of the roofs are each triangulated using six or seven tetrahedra (if the variable is unnegated or negated, resp.). Furthermore, the interface is triangulated by some vertices three times (in the positive case by $x_{1}^{i}$, by $x_{2}^{i}$, and by $z_{T}^{i}$ ) or two times (in the negative case by $\overline{x_{3}^{i}}$ and by $z_{T}^{i}$ ). The interface contains in each step between $C$ and $2 C$ triangles. Eventually, in either case there is one more tetrahedron (see above). An upper bound for the size of this triangulation is therefore

$$
\begin{aligned}
\# T & \leq(3 m+16)(C+V)+7 V+3 C V+1 \\
& =m(3 C+3 V)+\underbrace{16 C+23 V+3 C V+1}_{p_{T}(V, C)}
\end{aligned}
$$

What is the number of the vertices of the logical polytope in terms of the number of clauses and variables? We have $V$ logical variables and $C$ clauses in the SAT instance. Say we have $m$ interior points each of the vertex-edge chains we added (later we will replace the $m$ by a function of $V$ and $C$ ). We observe 
that we have $3 m+6$ vertices in each cupola, hence we have $(3 m+6)(V+C)$ for all cupolas. We have in each roof nine vertices, two of them are shared with the subsequent roof except for the last roof. Hence the total number of vertices in roofs is $7 V+2$. We have left only the $2 C+1$ vertices along the spine. In conclusion, the number of vertices of $P$ is

$$
\begin{aligned}
n & =(3 m+6)(V+C)+7 V+2+2 C+1 \\
& =m(3 C+3 V)+\underbrace{8 C+13 V}_{p_{n}(C, V)}+3
\end{aligned}
$$

We had said before that a "bad" triangulation (where at least one cupola skylight is triangulated by a vertex not lying in its visibility cone) has at least $n+m-3=m(3 C+3 V+1)+p_{n}(C, V)$ tetrahedra. On the other hand a "good" triangulation has at most $m(3 C+3 V)+p_{T}(C, V)$ tetrahedra. We can now set $m>p_{n}(C, V)-p_{T}(C, V)$ and $K=m(3 C+3 V)+p_{T}(C, V)$. Then, if a good triangulation exists, its size is smaller than or equal to $K$, and if not, all triangulations are larger than $K$. Note finally that the chosen $m$ and $K$ are polynomial in $C$ and $V$.

\subsection{Constructing the Logical Polytope}

Lemma 3.3 There is a polynomial algorithm that, given a logical formula on $V$ logical variables and $C$ logical clauses, produces a convex three-dimensional logical polytope as defined in Section 3.1. The coordinates of the vertices of the constructed polytope have binary encoding length polynomial in $V$ and $C$.

Proof: The construction will be carried out in five stages. By the time we end the construction all five requirements of the definition of the logical polytope must be satisfied, but three of the conditions will not be met until the last stage.

1. Give coordinates of the basic wedge, with rectangular faces on top for each variable.

2. Attach the roofs for each variable, giving preliminary coordinates for the literal vertices and preliminary coordinates for the points on the lower edge (the spine of the wedge).

3. Perturb the literal vertices to their final positions.

4. Perturb the vertices on the spine of the wedge.

5. Attaching the variable cupolas following the procedures of Section 2.

In every step we will build a construction element (a point, a line, or a plane) whose coordinates are polynomials in the construction elements up to that particular moment. Hence, the encoding length of each new construction element is bounded by a linear function of the encoding length of the construction so far. 
The number of construction steps is polynomially bounded in $C$ and $V$. Hence the encoding length of the whole construction is also polynomially bounded in $C$ and $V$. Note however, that the coordinates themselves will in general be exponentially large.

Instead of writing explicit (and highly cumbersome) coordinates for the construction elements, we rely on Lemma 1.4 to ensure that such coordinates can be found if one has really the desire to see a particular logical polytope. A key property of Stages 2-4 in the construction is that the geometric conditions we want to determine a finite collection of strict polynomial inequalities in a single variable. Then, by Lemma 1.4, we know there is an appropiate polynomial size solution. In subsequent stages of the construction similar new systems, for other independent parameters, will be solved, preserving what we had so far, but building up new properties.

Stage 1: The basic wedge. Consider the triangular prism which is the convex hull of the six points $c_{0}=(0,0,0), c_{2 C}=(0,1,0), z_{T}^{V}=(0,0,1), z_{F}^{1}=(1,0,1)$, $z_{L}^{V}=(0,1,1)$, and $z_{R}^{1}=(1,1,1)$. See Figure 21(a). In order to obtain a convex structure on the top of the wedge, we consider the function $f(x)=x(1-x)+1$. The vertices of each roof boundary (that is $z_{T}^{i}$ and $z_{F}^{i}$ as well as $z_{R}^{i}$ and $z_{L}^{i}$ ) will lie on the surface $z=f(x)$. More specifically, $z_{F}^{i}=z_{T}^{i+1}=(i / V, 0, f(i / V))$ and $z_{R}^{i}=y_{L}^{i+1}=(i / V, 1, f(i / V))$ for $i=0, \ldots, n$. By the concavity of $f$, the points are indeed in convex position and their convex hull, the wedge has the desired face lattice (see Figure 21(b)).
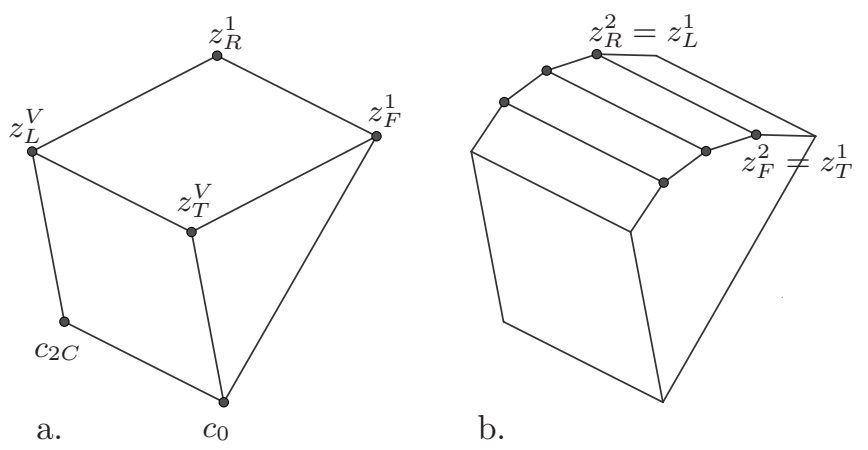

b.

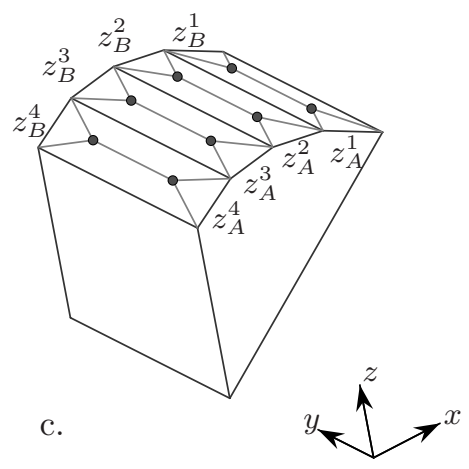

Figure 21: Construction of the wedge

So far none of the conditions we want are satisfied (not even partially).

Stage 2: The roofs. We will first attach the points $z_{A}^{i}$ and $z_{B}^{i}$ to the quadrilateral face $\left(z_{L}^{i}, z_{R}^{i}, z_{T}^{i}, z_{F}^{i}\right)$. Then we give preliminary coordinates to the literal vertices and to the vertices on the spine.

Let $z_{A}^{i}=1 / 2 \cdot\left(z_{T}^{i}+z_{F}^{i}\right)+\left(0,1 / 3, t_{\text {roof }}\right)$ and $z_{B}^{i}=1 / 2 \cdot\left(z_{T}^{i}+z_{F}^{i}\right)+\left(0,2 / 3, t_{\text {roof }}\right)$ where $t_{\text {roof }}$ is a non-negative parameter that is called the roof height. That is the points have the same $x$ coordinate as the midpoint between $z_{T}^{i}$ and $z_{F}^{i}, y$ coordinate $1 / 3$ and $2 / 3$ respectively, and height $t_{\text {roof }}$ over the face $\left(z_{T}^{i}, z_{F}^{i}, z_{L}^{i}, z_{R}^{i}\right)$. We want to choose $t_{\text {roof }}$ in a way that $z_{A}^{i}$ and $z_{B}^{i}$ are beyond the facet $\left(z_{T}^{i}, z_{F}^{i}, z_{L}^{i}, z_{R}^{i}\right)$ 
(see Figure 21(c)). We can easily achieve this by the technique presented in Lemma 1.4: The only possibly concave edges are the $\left(z_{T}^{i}, z_{L}^{i}\right)$. One restriction is therefore that all determinants $\operatorname{det}\left(z_{T}^{i}, z_{L}^{i}, z_{A}^{i-1}, z_{A}^{i}\right)$ have to be positive. These are finitely many open quadratic conditions on $t_{\text {roof }}$. For $t_{\text {roof }}=0$ the points $z_{A}^{i}$ an $z_{B}^{i}$ are inside the facets $\left(z_{T}^{i}, z_{F}^{i}, z_{L}^{i}, z_{R}^{i}\right)$, hence the edges in question are trivially convex. We will get more polynomial constraints on $t_{\text {roof }}$ below and then solve all simultaneously to find the suitable roof height.

The spine of the wedge is still a line. We now put preliminary points $c_{0}, \ldots, c_{2 C}$ on this line. Let

$$
u(j)=\frac{1}{2} \frac{j}{2 C}
$$

and $c_{j}=(0, u(j), 0)$ for $j=0, \ldots, 2 C-1$, and $c_{2 C}=(0,1,0)$ (see Figure 22). As an auxiliary point, let $b_{l}$ be the barycenter of the points $c_{2 l-2}, c_{2 l-1}$, and $c_{2 l}$ $(l=1, \ldots, C)$. At this moment, this point $b_{l}=c_{2 l-1}$. Later, as we perturb the spine vertices $b_{l}$ will move accordingly, always $b_{l}=1 / 3\left(c_{2 l-2}+c_{2 l-1}+c_{2 l}\right)$.

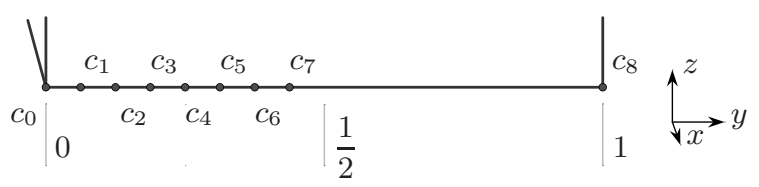

Figure 22: Preliminary coordinates for the spine vertices

Now we want to give initial positions to the literal vertices. Say variable $X_{i}$ occurs unnegated in clauses $l_{1}$ and $l_{2}$ and negated in $l_{3}$. Note that $l_{j}$ depend on the variable we are considering. For instance, in our example logical formula on p. 19, for variable $X_{1}, l_{1}=1, l_{2}=3$, and $l_{3}=2$. But for variable $X_{2}, l_{1}=2$, $l_{2}=3$, and $l_{3}=1$.

The preliminary literal vertex $x_{1}^{i}$ is the intersection of the $y=u\left(2 l_{1}-1\right)$ plane with the line connecting $z_{F}^{i}$ and $z_{B}^{i}$. We do the same for the other positive occurrence of $X_{i}$ and obtain the preliminary $x_{2}^{i}$. For the negative occurrence of $X_{i}$, we take the line connecting $z_{T}^{i}$ and $z_{B}^{i}$, intersect it with the $y=u\left(2 l_{3}-1\right)$ plane, and obtain the preliminary $\overline{x_{3}^{i}}$. We join the preliminary $x_{1}^{i}$ and $b_{l_{1}}$ by a line $d_{1}^{i}$ (this line lies in the $y=u\left(2 l_{1}-1\right)$ plane). Do the analogue process for $x_{2}^{i}$ and $\overline{x_{3}^{i}}$, obtaining $d_{2}^{i}$ and $d_{3}^{i}$. Later we will move the vertices $x_{1}^{i}, x_{2}^{i}, \overline{x_{3}^{i}}$ along their respective lines $d_{1}^{i}, d_{2}^{i}, d_{3}^{i}$ a little out of polytope in order to turn them into extreme points. The lines $d_{j}^{i}$ will also be used for blocking conditions.

Let $H^{i}$ be the plane that contains $z_{T}^{i}$ and $z_{F}^{i}$ and the midpoint of the edge $\left(z_{L}^{i}, z_{B}^{i}\right)$ (Figure 24). The only vertices above $H^{i}$ are $x_{1}^{i}, x_{2}^{i}, \overline{x_{3}^{i}}, z_{A}^{i}$, and $z_{B}^{i}$, and the only vertices on $H^{i}$ are $z_{T}^{i}$ and $z_{F}^{i}$. This follows from the convexity of the current polytope.

Let $g_{1}^{i}\left(g_{2}^{i}\right)$ be the line in the plane $H^{i}$ which is incident to $z_{F}^{i}$ and intersects the line $d_{1}^{i}\left(d_{2}^{i}\right)$. Note that this intersection point lies in the segment $\left(x_{1}^{i}, c_{2 l_{1}-1}\right)$ (the line segment $\left(x_{2}^{i}, c_{2 l_{2}-1}\right)$ ), thus in the interior of the constructed polytope. Analogously, let $g_{3}^{i}$ be the line in the plane $H^{i}$ which is incident to $z_{T}^{i}$ and 

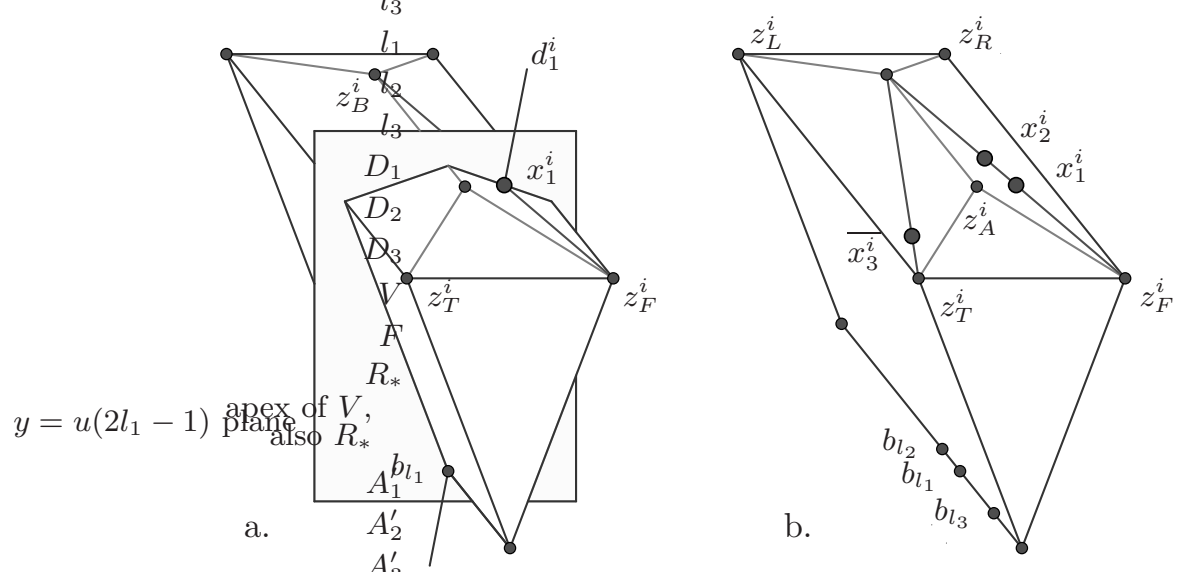

Figure 23: Construction of the literal vertices in the $X_{i}$ slice of the wedge

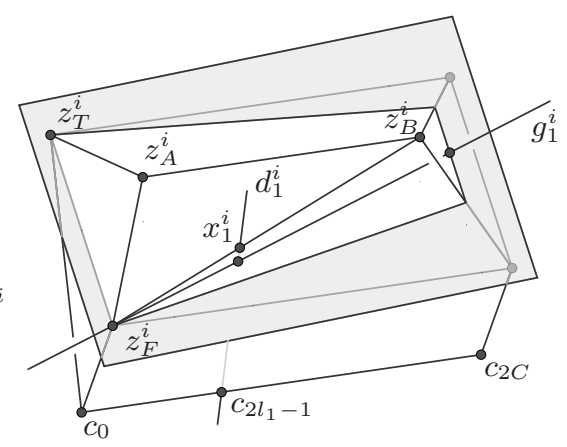

Figure 24: Construction of $H^{i}$ and $g_{1}^{i}$

intersects the line segment $\left(\overline{x_{3}^{i}}, c_{2 l_{3}-1}\right)$. It can be verified that if the roof height is small $\left(z_{L}^{i}, z_{R}^{i}, z_{B}^{i}\right)$ is pierced by the $g_{j}^{i}$ in its relative interior. This is another strict polynomial inequality in $t_{\text {roof. }}$. It will be the planes $H^{i}$ and lines $g_{j}^{i}(i=$ $1, \ldots, V)$ from which we make the visibility cones for the cupolas of variables $X_{i}$ according to Theorem 2.11.

It is important to note right now that the non-blocking conditions are satisfied for this special position of the vertices. We do not want the tetrahedron $\left(z_{T}^{i}, z_{L}^{i}, z_{R}^{i}, z_{B}^{i}\right)$ and the triangle $\left(x_{1}^{i}, c_{2 l_{1}-2}, c_{2 l_{1}}\right)$ to intersect. From this we get strict polynomial inequalities on $t_{\text {roof }}$. They are satisfied for $t_{\text {roof }}=0$ since the $y$ coordinates of the spine vertices $c_{l}$ are smaller than $1 / 2$. A suitable value of $t_{\text {roof }}$ can be found solving the univariate inequality system we accumulated in our discussion (Lemma 1.4). It is easy to check that the sweeping conditions are also satisfied for the preliminary position of the points $x_{1}^{i}, x_{2}^{i}, \overline{x_{3}^{i}}$. So far we have met two of the five required conditions to have a logical polytope.

Stage 3: Literal vertices Now we put the final $x_{j}^{i}(j=1,2,3)$ a little outward 
on line $d_{j}^{i}$ (Figure 23). A little for $x_{1}^{i}$ and $x_{2}^{i}$ means that the positive literal vertices lie in a plane parallel to the face $\left(z_{R}^{i}, z_{B}^{i}, z_{A}^{i}, z_{F}^{i}\right)$ very close to it. We treat $\overline{x_{3}^{i}}$ similarly. If the three literal vertices are moved a sufficiently small distance $t_{\text {literal }}$ the face lattice of what we get after taking the convex hull is as Figure 15 in all roofs.

By construction $H_{i}$ contains $z_{F}^{i}$ and $z_{T}^{i}$, and the $y=u(2 j-1)$ planes contain all literal vertices corresponding to clause $j$. This will become important for the visibility conditions (see Stage 5 ). Also, for small $t_{\text {literal }}$ the non-blocking and sweeping conditions are satisfied.

Although we do not have the blocking condition yet auxiliary lines can be set up: As above, let $l_{1}, l_{2}, l_{3}$ be the clauses to which the literal vertex $x_{1}^{i}$, $x_{2}^{i}, \overline{x_{3}^{i}}$ belong. We made sure that the line segments $\left(c_{2 l_{1}-1}, x_{1}^{i}\right)$ and $\left(z_{F}^{i}, z_{B}^{i}\right)$ intersect in their respective relative interiors. Hence, by the construction of line $g_{1}^{i}$, it is also pierced by $\left(x_{1}^{i}, c_{2 l_{1}-1}\right)$ between $z_{F}$ and the face $\left(z_{L}^{i}, z_{R}^{i}, z_{B}^{i}\right)$. (Analogously, $\left(c_{2 l_{2}-1}, x_{2}^{i}\right)$ and $\left(z_{F}^{i}, g_{2}^{i} \cap\left(z_{L}^{i}, z_{R}^{i}, z_{B}^{i}\right)\right)$ as well as $\left(c_{2 l_{3}-1}, \overline{x_{3}^{i}}\right)$ and $\left(z_{T}^{i}, g_{3}^{i} \cap\left(z_{L}^{i}, z_{R}^{i}, z_{B}^{i}\right)\right)$ intersect in their relative interiors). Later on this intersection will evolve into the real blocking conditions using Theorem 2.11.

Stage 4: The perturbing the vertices on the spine of the wedge. We now perturb the points $c_{j}$ on the spine of the wedge. Every even-indexed $c_{2 l}$ is changed to lie on a parabola, and for the moment the odd-indexed vertices $c_{2 l-1}$ are changed to lie on the line connecting $c_{2 l-2}$ and $c_{2 l}$. The $y$ coordinates of all points stay the same:

$$
c_{2 l}=\left(\frac{1}{2}(y-1)^{2} \cdot t_{\mathrm{even}}, y,(y-1)^{2} \cdot t_{\mathrm{even}}\right) .
$$

Note that by the $1 / 2$ in the $x$ coordinate, the points are moved into the polytope. The changes (parameter $t_{\text {even }}$ ) must be small enough that the convex hull now has the desired appearance (Figure 25) and the non-blocking conditions and the sweeping conditions are still satisfied. Once more we appeal to Lemma 1.4. The polynomials inequalities are now on the variable $t_{\text {even }}$ and the sweeping and non-blocking were satisfied at $t_{\text {even }}=0$. The reader should note that while the constructed vertices in the roofs do not change coordinates, dependent construction elements like the lines $d_{j}^{i}$ (connecting $x_{j}^{i}$ and $c_{2 l_{j}-1}$ ) and $g_{j}^{i}$ (lying in $H^{i}$ and intersecting $d_{j}^{i}$ ) change when the spine vertices move. However, the parameter $t_{\text {even }}$ has to be small enough that the preliminary blocking conditions are still met: $g_{j}^{i}$ still pierce the facet $\left(z_{L}^{i}, z_{R}^{i}, z_{B}^{i}\right)$ in its relative interior, and $g_{j}^{i}$ and $d_{j}^{i}$ intersect in the interior of the polytope.

Now we move the odd points $c_{2 l-1}$ beyond the face $G_{l}=\left(c_{2 l-2}, c_{2 l}, z_{T}^{0}\right)$ : to this end we choose a point $p_{l}$ beyond $G_{l}$ and move to $c_{2 l-1}+t_{\text {odd }}\left(p_{l}-c_{2 l-1}\right)$. Such a point $p_{l}$ is easily found by taking a normal to $G_{l}$ through its barycenter and moving outwards while staying beyond the face (note that this involves again Lemma 1.4, see the definition of beyond). The parameter $t_{\text {odd }}$ is chosen small enough: Convexity and the correctness of the face lattice are easily achieved. Also the sweeping conditions are valid for slight moves. Keeping $t_{\text {odd }}$ small also guarantees the non-blocking conditions: the tetrahedron $\left(x_{1}^{i}, c_{2 l_{1}-2}, c_{2 l_{1}-1}, c_{2 l_{1}}\right)$ 

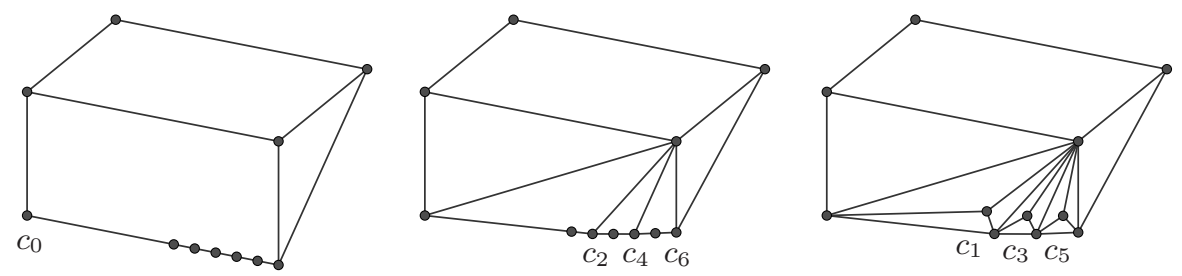

Figure 25: Perturbation of the vertices on the spine

is only slightly bigger than just the triangle $\left(x_{1}^{i}, c_{2 l_{1}-2}, c_{2 l_{1}}\right)$ which did not intersect the tetrahedron $\left(z_{T}^{i}, z_{L}^{i}, z_{R}^{i}, z_{B}^{i}\right)\left(x_{2}^{i}\right.$ and $\left.\overline{x_{3}^{i}}\right)$.

For the blocking conditions, let $X_{i}$ be the $j$ th logical variable in clause $l$. Note that now the line $d_{j}^{i}$ intersects the triangle $\left(c_{2 l-2}, c_{2 l-1}, c_{2 l}\right)$ in its relative interior. The lines $g_{j}^{i}$ are updated as the lines $d_{j}^{i}$ move. Since $t_{\text {odd }}$ is small, $g_{j}^{i}$ still pierces the facet $\left(z_{L}^{i}, z_{R}^{i}, z_{B}^{i}\right)$ in its relative interior, and $g_{j}^{i}$ and $d_{j}^{i}$ intersect in the interior of the polytope. Note that $d_{j}^{i}$ is still in the $y=u(2 l-1)$ plane because the $y$ coordinates of the spine vertices were conserved.

Stage 5: Attaching the cupolas. It remains to construct all the cupolas. Over the facets $\left(z_{L}^{i}, z_{R}^{i}, z_{B}^{i}\right)(i=1, \ldots, V)$ we construct cupolas using the planes $H_{i}$ and sets of lines $\left\{g_{1}^{i}, g_{2}^{i}, g_{3}^{i}\right\}$, and over the facets $\left(c_{2 l-2}, c_{2 l-1}, c_{2 l}\right)(i=1, \ldots, C)$ we construct the clause cupolas using the $y=g(2 l-1)$ planes and the sets of lines $\left\{d_{j}^{i} \mid X_{i}\right.$ 's $j$ th occurence is in clause $\left.l\right\}$. We invoke Theorem 2.11 and get the final polytope. By this construction, it is convex, has the correct face lattice, and the visibility conditions are satisfied.

The reader will recall that $g_{j}^{i}$ and $d_{j}^{i}$ intersect in the interior of the polytope. Say again variable $X_{i}$ occurs unnegated in clauses $l_{1}$ and $l_{2}$ and negated in $l_{3}$. By Theorem 2.11 $g_{j}^{i}$ pierces the skylight of the cupola corresponding to variable $X_{i}$ and $d_{j}^{i}$ pierces the skylight corresponding to its clause $l_{j}$. Hence, the tetrahedron spanned by $z_{F}^{i}$ and the variable $X_{i}$ 's skylight and the tetrahedron spanned by $x_{1}^{i}\left(x_{2}^{i}\right)$ and clause $l_{1}$ 's skylight ( $l_{2}$ 's skylight) intersect in their interiors. Analogously, the tetrahedron spanned by $z_{T}^{i}$ and the variable $X_{i}$ 's skylight and the tetrahedron spanned by $\overline{x_{3}^{i}}$ and clause $l_{3}$ 's skylight intersect in their interiors. These are exactly the blocking conditions.

All other conditions concerned only points we constructed before, so they are still satisfied. The final polytope is therefore a logical polytope.

Proof of Theorem 1.1: The problem is clearly in NP: checking whether a collection of tetrahedra is indeed a triangulation of the polytope $P$ needs only a polynomial number of calculations. Every pair of tetrahedra is checked for proper intersection (in a common face or not at all), and the sum of the volumes equals the volume of $P$ (computable for instance by the Delaunay triangulation of the polytope). Also the size of triangulations of a given polytope is bounded by a polynomial in $n$ of degree two (this follows from the well-known upper bound theorem, for details see [21]). 
By Lemma 3.3, from a given logical formula on $V$ logical variables and $C$ clauses, we can construct a logical polytope $P$ of encoding length polynomial in $V$ and $C$. Hence, by Lemma 3.2 there is a polynomial transformation that establishes the polynomial equivalence of a solution for the SAT problem and small triangulations of $P$. This completes the proof.

\section{Related Problems and Conclusions}

In this last section we discuss Corollary 1.2 stated in the introduction and relate our main theorem to previously known results in the literature of optimal triangulations about the size of triangulations. We conclude with some remarks.

Proof of Corollary 1.8: For part (1), consider the logical polytopes $P$ we have constructed. If we could find the size $s_{\min }$ for their minimal triangulations in polynomial time, either $s_{\min } \leq K$, in which case we have indeed a triangulation smaller or equal to $K$, or $K<s_{\min }$ in which case we can be sure there is no triangulation for the logical polytope of size $K$ or less. This proves it must be hard to find a minimal triangulation in dimension 3 . To extend to any other dimension simply note that pyramids over the logical polytopes have triangulations determined essentially by the logical polytope. In fact part (2) follows also from the pyramid construction because the logical polytope is a face.

It is known that the sizes of triangulations for a $d$-dimensional polytope with $n$ vertices lie between $n-d$ and $f_{d}(\partial C(n+1, d+1))-d-1$, where $f_{d}(\partial C(n+1, d+1))$ is the number of $d$-dimensional facets of a $d+1$ dimensional cyclic polytope with $n+1$ vertices [21]. In particular, for 3-polytopes the possible number of tetrahedra ranges from $n-3$ to $\left(\begin{array}{l}n \\ 2\end{array}\right)-2 n+3$. Both bounds are known to be tight for three dimensions 13]. It is also known that the size of a minimal triangulation of a convex 3-polytope must lie between $n-3$ and $2 n-10$, when $n>12$ [13]. That the upper bound is tight was proved in [25] using hyperbolic geometry. It is worth noticing at this point that the size of the constant $K$ we constructed in the previous section satisfies in fact $n-3<K<2 n$. Now we discuss an interesting reason why the lower bound is strict:

We say that a convex polytope is stacked if it has a triangulation whose dual graph is a tree (the dual graph of a simplicial complex is the graph that has one vertex for each maximal-dimensional simplex and two vertices are connected precisely when the corresponding simplices are adjacent via a common facet). The reader should be aware that in the literature the terminology stacked polytope is often restricted to simplicial polytopes. Here of course, we use it allowing that the stacking of simplices may give coplanarities. For example, any 3 -cube or triangular prism is a stacked polytope under our definition.

It turns out that a convex $d$-polytope $P$ with $n$ vertices has a triangulation of size $n-d$ precisely when $P$ is a stacked polytope (see [21]). It is natural to ask which polytopes are stacked. If it were NP-hard to recognize stacked polytopes then this would provide another proof that the problem of finding minimal triangulations is also in the same class. However we can prove: 
Theorem 4.1 1. For a convex d-polytope $P$ with $n$ vertices there is a $O\left(n^{d+2}\right)$ algorithm to decided whether $P$ is stacked (i.e., $P$ has a triangulation with $n-d$ maximal simplices). The algorithm uses only the 1 skeleton of the polytope. In particular, the size of a minimal triangulation of a stacked polytope does not depend on the particular coordinatization, but only depends on its face lattice.

2. A convex 3-dimensional polytope $P$ is stacked if and only if its graph does not contain as a minor the graph of an octahedron or a pentagonal prism.

Proof: We need some definitions that have been introduced earlier in the graph theory literature 11, 2, 12]. We say a graph $G$ is $k$-decomposable if $G$ has $k+1$ or fewer vertices or there is a subset of vertices $S$ of $G$ with at most $k$ vertices such that 1) $S$ is a cut, i.e. $G-S$ is disconnected, and 2) each of the connected components of $G-S$ has the property that when the vertices of $S$ are added back together with the complete graph on those vertices, the resulting graph is again $k$-decomposable.

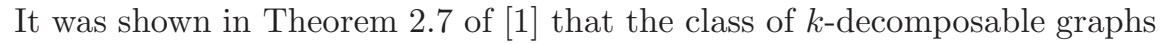
is the same as the class of partial $k$-trees: a graph is a $k$-tree if it can be reduced to the complete graph $K_{k}$, by a finite sequence of removals of degree $k$ vertices with completely connected neighbors (i.e. neighbors of the vertex induce a complete graph $K_{k}$ ). A partial $k$-tree is simply an edge-subgraph of a $k$-tree.

Now we claim that a $d$-dimensional convex polytope $P$ is stacked, if and only if its 1-skeleton is a partial $d$-tree. Here is the proof: the "only if" implication is clear from the definition of stacked polytope. We can prove the "if" implication by induction on $n$. The theorem is trivial if $n=d+1$ because then $P$ is a simplex and its graph is a complete graph, thus is a $d$-tree. Assume then $n>d+1$ and that the result is true for polytopes with fewer than $n$ vertices. Remember that if 1-skeleton $G(P)$ is a partial $d$-tree then it is $d$-decomposable. Thus there is a cut $S$ of cardinality at most $d$. The set $S$ must have in fact cardinality $d$ because $G(P)$ is $d$-connected by Balinski's theorem. If one has a vertex-cutset $S$ of cardinality $d$ in $G(P)$, then the hyperplane $H(S)$ spanned by $S$ intersects $G(P)$ only in the vertices of $G(P) \cap S$ and in no edges (otherwise $S$ is not a cut). In conclusion, $H(S) \cap P$ is a $(d-1)$-simplex and because $G(P)$ is $d$-decomposable we can apply induction hypothesis to prove the polytopes $H(S)^{+} \cap P$ and $H(S)^{-} \cap P$ are $d$-decomposable, and thus they are partial $d$ trees with fewer vertices than $P$, so both polytopes are stacked. Finally, note that their stacked triangulations match well at the common boundary simplicial facet $H(S) \cap P$, proving that $P$ is stacked.

Arnborg et al. discuss in [2] an algorithm that, for fixed values of $k$, decides whether a given graph is a partial $k$-tree. The total running time is $O\left(n^{k+2}\right)$. We also know that computing the 1-skeleton of a $k$-polytope can be done with the same complexity. This, together with the above claim completes the proof of the first part of the theorem.

For the second part we observe that partial $k$-trees form a minor closed family. This means that the set of partial $k$-trees is closed under taking edgedeletion or edge-contraction operations. The famous results of [24] imply that 
they can be characterized by a finite set of forbidden minors. El-mallah and Colbourn [12 proved that a graph is a planar 3-tree if and only if it has no minor isomorphic to the graph of an octahedron or a pentagonal prism. This fact together with our claim complete the proof of the second part.

\section{Remarks:}

1) The "coning" triangulation proposed in 13 provides an algorithm which is polynomial on the number of vertices and gives a 2-approximation of the minimal triangulation as it produces a triangulation of size less than or equal to $2 n-7$.

2) Given a 3-dimensional convex polytope $P$, a proper subset $S$ of tetrahedra with vertices in vertices $(P)$, and a positive integer $K$. Deciding whether there is a triangulation of $P$ that uses $K$ simplices from $S$ can also be proved to be an NP-hard problem using the constructions we explained.

3) It is interesting to note that the constructions presented in [6 prove also that covering a convex 3-polytope with tetrahedra can be done with fewer pieces than triangulating. A cover is a collection of simplices whose union is the whole polytope, but the elements can intersect in their interiors. It is would be interesting to know what is the computational complexity of finding minimal simplicial covers. Another interesting question would be what is the complexity of deciding whether a triangulation of the boundary of convex non-simplicial 3polytope extends to a triangulation of the whole polytope without adding new interior points? If hard, this could be used to establish another proof our results. The curious reader can easily prove that already for a triangular prism not all triangulations of the boundary extend to a triangulation of the whole polytope. Even more interesting. The triangulations of the boundary of a 3-cube extend or not depending on the coordinates of its vertices.

\section{References}

[1] Arnborg S. and Proskurowski A. Characterization and recognition of partial 3-trees SIAM J. Algebraic and Discrete Meth., 7, No 2, 1 986, 305314.

[2] Arnborg S., Corneil D. G. and Proskurowski A. Complexity of finding embeddings in a $k$-tree SIAM J. Algebraic and Discret Meth.,8, No. 2, 1987, 277-284.

[3] Arnborg S. and Proskurowski A . Linear time algorithms for NP-hard problems restricted to partial $k$-trees Discrete Applied Math., 23, 1989, 1124 .

[4] Arnborg S., Proskurowski A. and Corneil D.G. Forbidden minors characterizations of partial 3-trees Discrete Math., 80, 1990, 1-19.

[5] Avis D. and ElGindy H. Triangulating point sets in space, Discrete Comput. Geom., 2, 1987, 99-111. 
[6] Below A., Brehm U., De Loera J.A., and Richter-Gebert J. Minimal Simplicial Dissections and Triangulations of Convex 3-Polytopes, Discrete Comput. Geom., 24, 2000, 35-48.

[7] Bern M. and Eppstein D. Mesh generation and optimal triangulation, in Computing in Euclidean Geometry (D.Z Du and F.K Hwang eds.), World Scientific Publishing Co. 1992 (see also second edition appeared in 1995).

[8] Billera L. and Björner A. Face numbers of polytopes and complexes. in Handbook of Discrete and Computational Geometry, 271-290, (Goodman J.E. and O'Rourke J. eds.), CRC Press, New York,

[9] Björner A., Las Vergnas M., Sturmfels B., White N., and Ziegler G.M. Oriented Matroids, Cambridge University Press, Cambridge, 1992.

[10] Chazelle B. and Palios L., Triangulating a Nonconvex Polytope, Discrete Comput. Geom., 5, 1990, 505-526.

[11] Cottle R.W. Minimal triangulation of the 4-cube, Discrete Mathematics, 40, 1982, 25-29.

[12] El-Mallah E. and Colbourn C.J On two dual classes of planar graphs. Discrete Math., 80, 1990, 21-40.

[13] Edelsbrunner H., Preparata F. P., and West D. B. Tetrahedrizing point $s$ ets in three dimensions, Journal of Symbolic Computation, 10, 1990, $335-347$.

[14] Garey M.R. and Johnson S.J. Computers and Intractability: A Guide to the Theory of NP-Completeness, Freeman, San Francisco, 1979.

[15] Haiman M. A simple and relatively efficient triangulation of the $n$-cube Discrete Comput. Geom., 6, 1991, 287-289.

[16] Hughes R.B., Anderson M. R. Simplexity of the cube, Discrete Math., 158, 1996, 99-150.

[17] Lee C.W. Subdivisions and triangulations of polytopes in Handbook of Discrete and Computational Geometry, 271-290, (Goodman J.E. and O'Rourke J. eds.) , CRC Press, New York, 1997.

[18] Lee C.W. Regular triangulations of convex polytopes, Applied Geometry and Discrete Mathematics- The Victor Klee Festschrift (P. Gritzmann and B. Sturmfels eds.) DIMACS series in Discrete Math. and Theoretical Comp. Science, 4, 1991, 443-456.

[19] O'Rourke J. Art Gallery Theorems and Algorithms, Oxford Univ. Press, New York/Oxford, 1987.

[20] Richter-Gebert, J. Finding small triangulations of polytope boundaries is hard, to appear in Discrete and Comput. Geom. 
[21] Rothschild G.L. and Straus E.G. On triangulations of the convex hull of $n$ points, Combinatorica, 5, 1985, 167-179.

[22] Ruppert J. and Seidel R. On the difficulty of triangulating threedimensional non-convex polyhedra, Disc. Comp. Geom., 7, 1992, 227-253.

[23] Schönhardt E. Über die Zerlegung von Dreieckspolyedern in Tetraeder, Math. Ann., 98, 1928, 309-312.

[24] Robertson N. and Seymour P.D. Graph minors-a survey in Surveys in Combinatorics, I. Anderson (editor), Cambridge University Press, 1985.

[25] Sleator, D.D., Tarjan R.E., and Thurston W.P. Rotation distance, triangulations, and hyperbolic geometry. J. Amer. Math. Soc., 1, 1988, 647681.

[26] Sturmfels B. Polynomial equations and convex polytopes Amer. Math. Monthly, 105, 1998, 907-922.

[27] Todd M. J. The Computation of Fixed Points and Applications, Volume 124 of Lecture Notes in Econom. a nd Math. Systems, Springer-Verlag, Berlin, 1976.

[28] Ziegler G.M. Lectures on Polytopes, Springer Verlag, New York, 1994. 


$$
\text { A }
$$

